\title{
ENUMERATION OF LATTICE POLYTOPES BY THEIR VOLUME
}

\author{
GABRIELE BALLETTI
}

\begin{abstract}
A well known result by Lagarias and Ziegler states that there are finitely many equivalence classes of $d$-dimensional lattice polytopes having volume at most $K$, for fixed constants $d$ and $K$. We describe an algorithm for the complete enumeration of such equivalence classes for arbitrary constants $d$ and $K$. The algorithm, which gives another proof of the finiteness result, is implemented for small values of $K$, up to dimension six. The resulting database contains and extends several existing ones, and has been used to correct mistakes in other classifications. When specialized to three-dimensional smooth polytopes, it extends previous classifications by Bogart et al., Lorenz and Lundman. Moreover, we give a structure theorem for smooth polytopes with few lattice points that proves that they have a quadratic triangulation and that we use, together with the classification, to describe smooth polytopes having small volume in arbitrary dimension. In dimension three we enumerate all the simplices having up to 11 interior lattice points and we use them to conjecture a set of sharp inequalities for the coefficients of the Ehrhart $h^{*}$-polynomials, unifying several existing conjectures. Finally, we extract and discuss minimal interesting examples from the classification, and we study the frequency of properties such as being spanning, very ample, IDP, and having a unimodular cover or triangulation. In particular, we find the smallest polytopes which are very ample but not IDP, and with a unimodular cover but without a unimodular triangulation.
\end{abstract}

\section{INTRODUCTION}

Finiteness results are not uncommon in the study of lattice polytopes. Most of these are proven by fixing the dimension, showing an upper bound for the volume and then using the following result by Lagarias and Ziegler.

Theorem 1.1 ([LZ91, Theorem 2]). Up to unimodular equivalence, there are finitely many d-dimensional lattice polytopes having volume lower than a constant $K$.

Note that working up to unimodular equivalence, i.e. up to affine lattice preserving maps in $G L_{d}(\mathbb{Z}) \times \mathbb{Z}^{d}$ is an obvious requirement that we will often avoid to mention.

Once it is known that a family of lattice polytopes is finite, it is tempting to give a complete description of it. Most of the times this seems not to be possible in full generality, and it is instead done explicitly only fixing "small enough" parameters, first and foremost the dimension. A well-known example of finiteness result is the finiteness of $d$-dimensional lattice polytopes having a fixed positive number of interior lattice points, which follows from a volume bound proven by Hensley Hen83. This result paved the way to explicit classifications of families of lattice polytopes having a fixed number of interior lattice points. The best example is probably the massive classification of reflexive

2010 Mathematics Subject Classification. 52B20 (Primary); 52B11 (Secondary).

Key words and phrases. Lattice polytopes; enumeration; classification; volume. 
polytopes (which have one interior lattice point) up to dimension four performed by Kreuzer and Skarke to study mirror symmetric Calabi-Yau manifolds [KS98, KS00. Another example of polytopes having exactly one interior point are the smooth Fano polytopes, fully enumerated up to dimension nine [Bat81, WW82, Bat99, Sat00, KN09, Øb07, LP08. Without additional restrictions, lattice polytopes having one and two interior lattice points are classified in dimension three [Kas10, BK16].

In the last years, many other examples of these kind of results were proven. In AWW11, AKW17] is proven that in each dimension there are finitely many hollow lattice polytopes which are maximal up to inclusion, and they are classified in dimension three. In $\left[\mathrm{BHH}^{+} 15\right]$ the finiteness of smooth polytopes having fixed number of lattice points is shown in each dimension. Such polytopes are enumerated in dimension three, up to 16 lattice points [Lor10, Lun13]. A finiteness result in dimension three for polytopes of width larger than one and fixed number of interior points is proven in [BS16a], and an explicit enumeration has been performed up to 11 lattice points [BS16b, BS17].

All the aforementioned results are proven via bounding the volume of the considered family of polytopes and applying Theorem 1.1. In this paper we take a natural step, and use Lagarias and Ziegler's Theorem to perform a systematic enumeration of all lattice polytopes of fixed dimension and volume that are within computational reach. This is done by giving an alternative proof of Theorem 1.1 that has the advantage of being efficiently implementable. The key is to build the polytopes "from below", starting from a simplex and progressively adding vertices, instead of "from above" as the original proof of Theorem 1.1 suggest, carving out all the possible lattice polytopes from a big cube.

A result with a similar taste, but only in dimension two, has been achieved by Castryck in Cas12. With a "moving out" technique, he gives a different proof of a finiteness result and classifies all lattice polygons having up to 30 interior lattice points.

The paper has the following structure. In Section 2 we give an introduction to point configurations, building the necessary tools for the rest of the paper. In Section 3 we give an independent proof of Theorem 1.1. which leads to an algorithm, whose implementation is discussed in Section 4. In Section 5 we discuss the results of the classification and we compare it with existing ones. In Section 6 we specialize the classification to smooth polytopes. In this settings our classification extends other ones performed in dimension three, and creates a database of small smooth polytopes. We give a structure theorem for $d$-dimensional smooth polytopes having at most $3 d-4$ lattice points (Theorem 6.10), which we immediately apply to fully categorize smooth polytopes having normalize volume at most 10 (Proposition 6.14). In Section 7 we use the classification together with existing volume bounds for simplices with a fixed number of interior lattice points to classify all the three-dimensional lattice simplices having up to 11 interior lattice points (Corollary 7.5). In this section, we use the classification of the precedent section to give conjectural Ehrhart inequalities for three-dimensional lattice polytopes (Conjecture 8.7). In the final Section 9, we look for interesting examples contained in the database, and we observe the commonness of polytopes which are spanning, very ample, IDP, have a unimodular cover/triangulation (see Appendix C).

This project originated from the following question that Christian Haase posed for the participants of the "Workshop on Convex Polytopes for Graduate Students" in Osaka, during January 2017. 
"How many 6-dimensional lattice polytopes of volume 5 have the integerdecomposition property?"

From the tables in Appendix C we can read that the answer is 27.

I would like to thank my PhD advisor Benjamin Nill for all the inspiring and patient discussions. The idea of Algorithm 2 came out during one of those. I am also grateful to Al Kasprzyk for teaching me how to use Magma and Paco Santos for helpful remarks. The author is partially supported by the Vetenskapsrådet grant NT:2014-3991

\section{INVITATION TO POINT CONFIGURATIONS AND VOLUME VECTORS}

In this section we sketch some basic concepts and results regarding point configurations, triangulations and volume vectors of lattice polytopes. This material will be used in Section 3 for proving Theorem 1.1. We use [DLRS10, Chapter 4] as a reference, but we also refer to [LZ91, Chapter 6] for details.

A point configuration is a finite set of points $\mathcal{A}$ in an affine space $\mathbb{R}^{d}$. We say that a point configuration $\mathcal{A}$ is independent if none of its points is an affine combination of the rest, otherwise we say that $\mathcal{A}$ is dependent. A point configuration has corank one if it has a unique (up to scalar multiplication) dependence relation $\sum_{\mathbf{p} \in \mathcal{A}} \lambda_{\mathbf{p}} \mathbf{p}=\mathbf{0}$. Such dependence relation defines a partition of $\mathcal{A}$ in the sets

$$
J_{+}:=\left\{\mathbf{p} \in \mathcal{A}: \lambda_{\mathbf{p}}>0\right\}, \quad J_{0}:=\left\{\mathbf{p} \in \mathcal{A}: \lambda_{\mathbf{p}}=\mathbf{0}\right\}, \quad J_{-}:=\left\{\mathbf{p} \in \mathcal{A}: \lambda_{\mathbf{p}}<\mathbf{0}\right\} .
$$

Such partition is unique, up to switching $J_{+}$with $J_{-}$. Given any point configuration $\mathcal{A}$ one can consider the polytope $P$ defined as the convex hull of the points of $\mathcal{A}$, i.e $P_{\mathcal{A}}:=\operatorname{conv}(\mathcal{A})$. If $\mathcal{A}$ has corank one then $P_{\mathcal{A}}$ has exactly two different triangulations in simplices having vertices on $A$.

Lemma 2.1 (DLRS10, Lemma 2.4.2]). If a point configuration $\mathcal{A}$ has corank one, then the following are the only two triangulations of $P_{\mathcal{A}}$ in simplices having vertices in $\mathcal{A}$ :

$$
\mathcal{T}_{+}:=\left\{C \subset \mathcal{A}: J_{+} \nsubseteq C\right\}, \quad \text { and } \quad \mathcal{T}_{-}:=\left\{C \subset \mathcal{A}: J_{-} \nsubseteq C\right\} .
$$

Note that, supposing $P_{\mathcal{A}}$ full-dimensional, the full-dimensional simplices in $\mathcal{T}_{+}$and $\mathcal{T}_{-}$ are $\left|J_{+}\right|$and $\left|J_{-}\right|$respectively, i.e. the signature of $\mathcal{A}$ is the pair of number of simplices of the two ways to triangulate $P_{\mathcal{A}}$ discussed above. If the unique dependence relation of a corank one point configuration $\mathcal{A}$ is entirely supported on $\mathcal{A}$, i.e. if $J_{0}$ is empty, then we say that $\mathcal{A}$ is a circuit. In particular every proper subset of a circuit is an independent point configuration. If $\mathcal{A}$ is a circuit the pair $\left(J_{+}, J_{-}\right)$is classically called the Radon partition or the oriented circuit of $\mathcal{A}$.

Being interest in lattice polytopes, we move our focus to point configurations contained in the lattice $\mathbb{Z}^{d}$. Given a full-dimensional lattice polytope $P$ in $\mathbb{R}^{d}$, we denote by $\operatorname{Vol}(P)$ its normalized volume $\operatorname{Vol}(P):=d ! \operatorname{vol}(P)$, where $\operatorname{vol}(P)$ is the standard euclidean volume. In other words we set the normalized volume of any lattice simplex $S$ with vertices $\mathbf{v}_{1}, \ldots, \mathbf{v}_{d+1}$ to be

$$
\operatorname{Vol}(S):=\left|\operatorname{det}\left(\begin{array}{ccc}
1 & \ldots & 1 \\
\mathbf{v}_{1} & \ldots & \mathbf{v}_{d+1}
\end{array}\right)\right|,
$$

Then the notion of volume can be extended to arbitrary polytopes via triangulations. The notion of volume can be extended in a finer way to polytopes, via volume vectors. For this we agree with the notation used in [BS16a]. 
Definition 2.2. Let $\mathcal{A}=\left\{\mathbf{p}_{1}, \ldots, \mathbf{p}_{n}\right\}$ in $\mathbb{Z}^{d}$, with $n \geqslant d+1$. Then the volume vector of $\mathcal{A}$ is defined as

where

$$
\left(w_{i_{1}, \ldots, i_{d+1}}\right)_{1 \leqslant i_{1}<\cdots<i_{d+1} \leqslant n} \in \mathbb{Z}^{\left(\begin{array}{c}
n \\
d+1
\end{array}\right)}
$$

$$
\left(w_{i_{1}, \ldots, i_{d+1}}\right):=\operatorname{det}\left(\begin{array}{ccc}
1 & \ldots & 1 \\
\mathbf{p}_{i_{1}} & \ldots & \mathbf{p}_{i_{d+1}}
\end{array}\right) .
$$

Note that we are assuming that $\mathcal{A}$ has an intrinsic order on the elements. The volume vector is a powerful invariant, which almost encodes all the data of a point configuration.

Proposition 2.3 ([BS16a, Proposition 2.2]). Let $\mathcal{A}$ and $\mathcal{A}^{\prime}$ be the point configurations $\mathcal{A}=\left\{\mathbf{p}_{1}, \ldots, \mathbf{p}_{n}\right\}$ and $\mathcal{A}^{\prime}=\left\{\mathbf{p}_{1}^{\prime}, \ldots, \mathbf{p}_{n}^{\prime}\right\}$ in $\mathbb{Z}^{d}$, and suppose that (with respect to a given

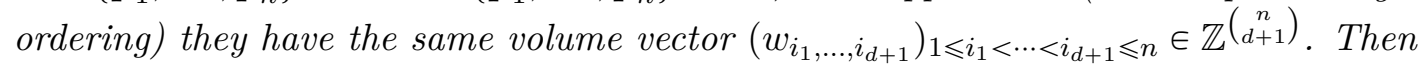

(1) There is a unique unimodular affine map $t: \mathbb{R}^{d} \rightarrow R^{d}$ with $t(\mathcal{A})=\mathcal{A}^{\prime}$ (respecting the order of points).

(2) If $\operatorname{gcd}_{1 \leqslant i_{1}<\cdots<i_{d+1} \leqslant n}\left(w_{i_{1}, \ldots, i_{d+1}}\right)=1$, then $t$ is a $\mathbb{Z}$-equivalence between $\mathcal{A}$ and $\mathcal{A}^{\prime}$. In particular $P_{\mathcal{A}}$ and $P_{\mathcal{A}^{\prime}}$ are unimodular equivalent lattice polytopes.

We now restrict our interest to point configurations in $\mathbb{Z}^{d}$ having $d+2$ elements. We always assume that the point configuration is full-dimensional, i.e. it affinely spans $\mathbb{R}^{d}$. Note that this is equivalent to assume the configuration to have corank one. In this case, we can simplify, and modify slightly, the notation for the entries of the volume vector. If $\mathcal{A}=\left\{\mathbf{p}_{1}, \ldots, \mathbf{p}_{d+2}\right\}$, then we denote the volume vector of $\mathcal{A}$ as

$$
w_{\mathcal{A}}=\left(w_{1}, \ldots, w_{d+2}\right), \text { where } w_{i}:=(-1)^{i+1} w_{1, \ldots, \hat{i}, \ldots, d+2} .
$$

The change of sign allows to simplify the statement in the following lemma.

Lemma 2.4 ([BS16a, Equation (2)]). Let $\mathcal{A}=\left\{\mathbf{p}_{1}, \ldots, \mathbf{p}_{d+2}\right\}$ be a corank one point configuration. Then its volume vector

$$
w_{\mathcal{A}}=\left(w_{1}, \ldots, w_{d+2}\right)
$$

sums up to zero and encodes the unique linear relation in $\mathcal{A}$ :

$$
\sum_{i=1}^{d+2} w_{i} \mathbf{p}_{i}=\mathbf{0}, \quad \text { and } \quad \sum_{i=1}^{d+2} w_{i}=0 .
$$

One may think the equality of $\sum_{i=1}^{d+2} w_{i}=0$ in the following way. The $w_{i}$ 's which are positive are the normalized volumes of the full dimensional simplices in $\mathcal{T}_{+}$, while the negative $w_{i}$ 's equal to (minus) the normalized volumes of the full-dimensional simplices in $\mathcal{T}_{-}$. The equality follows by noting that $\mathcal{T}_{+}$and $\mathcal{T}_{-}$are both triangulations of the same polytope. This is clarified by the following example.

Example 2.5. Let $\mathcal{A}$ be the point configuration given by the columns of the matrix below.

$$
\left[\begin{array}{lllll}
0 & 1 & 1 & 0 & 1 \\
0 & 1 & 0 & 1 & 1 \\
0 & 0 & 1 & 1 & 1
\end{array}\right]
$$

Then, $\mathcal{A}$ is the set of the vertices of the polytope $P_{\mathcal{A}}$, depicted below. 


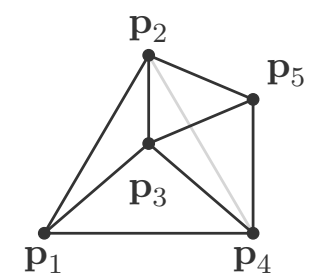

The volume vector of $\mathcal{A}$ is $w_{\mathcal{A}}=(1,-1,-1,-1,2)$. Note that the positive entries $(1,2)$ in $w_{\mathcal{A}}$ corresponds exactly to the normalized volumes of the two tetrahedra in $\mathcal{T}_{+}$, while the negative entries $(-1,-1,-1)$ corresponds exactly to (minus) the volumes of the three tetrahedra in $\mathcal{T}_{-}$.

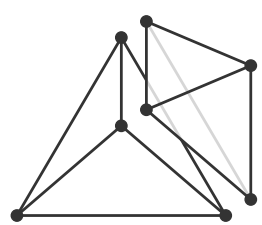

$\mathcal{T}_{+}$

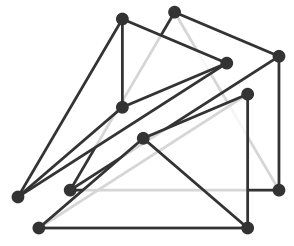

$\mathcal{T}_{-}$

In particular the entries of $w_{\mathcal{A}}$ sum to zero. We can furthermore check that they encode the unique affine linear relation in $\mathcal{A}$, indeed

$$
\mathbf{p}_{1}-\mathbf{p}_{2}-\mathbf{p}_{3}-\mathbf{p}_{4}+2 \mathbf{p}_{5}=\mathbf{0}
$$

\section{An implementable proof for the Lagarias-Ziegler Theorem}

In this section we give an alternative algorithmic proof to Theorem 1.1. Such algorithm will be then implemented for a complete enumeration of lattice polytopes with "reasonably small" volume and dimension, which is described in the following sections.

The original proof given in [LZ91 is divided in two parts, one proving the result for simplices, another extending it to polytopes. The "simplicial" part of the result is easily deduced by putting the matrix of the vertices of a simplex in a normal form. This part of the proof, as given in [LZ91, can be easily implemented, so we can use it as the first step of the algorithm, adding only some small improvements (see Algorithm 1). For the convenience of the reader we quickly sketch the theoretical argument used.

Lemma 3.1. There are finitely many equivalence classes of d-dimensional lattice simplices having volume lower than a constant $k$.

Proof. Let $S$ be the $d$-dimensional lattice simplex with vertices $\mathbf{v}_{0}, \mathbf{v}_{1}, \ldots, \mathbf{v}_{d+1}$. We can suppose $v_{0}$ to be the origin of the lattice. In this way $\operatorname{vol}(S)=|\operatorname{det}(M)|$, where $M$ is the $d \times d$ matrix whose columns are the vertices $\mathbf{v}_{1}, \ldots, \mathbf{v}_{d+1}$. We now take $M$ to the 
upper-triangular form (Hermite normal form)

$$
M^{\prime}:=\left(\begin{array}{cccc}
v_{1,1} & v_{1,2} & \cdots & v_{1, d} \\
& v_{2,2} & \cdots & v_{2, d} \\
& & \ddots & \vdots \\
& & & v_{d, d}
\end{array}\right),
$$

where on the $j$-th column $0 \leqslant a_{i, j}<a_{j, j}$ for $i=1, \ldots, j$, and $a_{i, j}=0$ for $i=j+1, \ldots, d$. Since the simplex $S^{\prime}$ whose vertices are the origin and the columns of $M^{\prime}$ is affinely equivalent to $S, \operatorname{Vol}(S)=\prod_{i=1}^{d} a_{i, i}$. Since all the entries of $M^{\prime}$ are positive, there are finitely many possible values for all the entries $a_{i, i}$, and consequently, for all the entries of $M^{\prime}$.

From now on, our proof diverges from the original one. In particular, we now focus our attention on the case of $d$-dimensional polytopes having $d+2$ vertices. We prove the result for this special case using the theory developed in the previous section and then we deduce the general case as a corollary.

Proposition 3.2. There are finitely many equivalence classes of d-dimensional lattice polytopes having $d+2$ vertices and volume lower than a constant $K$.

Proof. Let $P$ be a lattice polytopes with $d+2$ vertices such that $\operatorname{Vol}(P)<K$. We call $\mathcal{A}$ the point configuration given by the vertices of $P=P_{\mathcal{A}}$, it has corank one. Let $w_{\mathcal{A}}$ be the volume vector of $\mathcal{A}$. Since the volume of $P$ is bounded by $K$, the sum of the positive entries of $w_{\mathcal{A}}$ is $K$ at most. Similarly, the sum of the negative entries in $w_{\mathcal{A}}$ is $-K$ at least. In particular there are finitely many possible volume vectors vectors that can be the volume vector of $\mathcal{A}$. By Lemma 2.4 the previous statement means there are finitely many possible dependence relations which can be (up to multiplication by a scalar factor) the only dependence relation on $\mathcal{A}$. This proves that, if $S$ is any $d$-dimensional lattice simplex with $\operatorname{Vol}(S)<K$, then that the set

$$
\left\{\mathbf{p} \in \mathbb{Z}^{d}: \operatorname{Vol}(\operatorname{conv}(S \cup\{\mathbf{p}\})) \leqslant K\right\}
$$

is finite. Finally, we note that $P$ is completely determined by the choice of $d+1$ affinely independent and ordered vertices, plus the unique linear relation among its $d+2$ vertices. The convex hull of $d+1$ affinely independent vertices of $P$ is a $d$-dimensional simplex having normalized volume strictly smaller than $K$. By Lemma 3.1. there are finitely many such simplices.

From this we are able to prove Lagarias-Ziegler Theorem.

Proof of Theorem 1.1. A $d$-dimensional lattice polytope $P$ of volume $\operatorname{Vol}(P) \leqslant K$ has at most $K+d$ lattice points. This can be immediately deduced using some basic Ehrhart Theory (see Proposition 8.1). In particular, its number of vertices $n$ is at most $K+d$. Suppose the vertices of $P$ are ordered such that $\mathbf{v}_{0}, \ldots, \mathbf{v}_{d}$ are affinely independent. Then

$$
P=\operatorname{conv}\left(\bigcup_{i=d+2}^{n} P_{i}\right),
$$


where $P_{i}:=\operatorname{conv}\left(\mathbf{v}_{0}, \ldots, \mathbf{v}_{d}, \mathbf{v}_{i}\right)$ with $d+2 \leqslant i \leqslant n$. For each $i, P_{i}$ is a $d$-dimensional polytope with $d+2$ vertices, while $S:=\operatorname{conv}\left(\mathbf{v}_{0}, \ldots, \mathbf{v}_{d}\right)$ is a $d$-dimensional simplex. Since $\operatorname{Vol}(S) \leqslant \operatorname{Vol}\left(P_{i}\right) \leqslant \operatorname{Vol}(P) \leqslant K$, we conclude by Lemma 3.1 and Proposition 3.2 .

\section{IMPLEMENTATION}

In this section we describe the implementation of the results described in the previous section. Note that both the proofs of Theorem 1.1 for simplices and for polytopes admit a straightforward algorithmic implementation. Anyway, in order to have feasible running times, we are going to optimize the algorithms with some careful tweaking. Results of such implementation will be described in Section 5 .

We denote by $\mathcal{P}_{V}^{d}$ and $\mathcal{S}_{V}^{d}$ the sets of $d$-dimensional polytopes and simplices having normalized volume $V$. Note that, as usual, these sets are considered up to unimodular equivalence. Computationally speaking, this is not a problem: each polytope can be indeed put in a normal form, and $\mathcal{P}_{V}^{d}$ can be thought as the set of these forms.

Algorithm 1 fully enumerates all the elements of $\mathcal{S}_{V}^{d}$. To speed things up, we can "recycle" the enumeration of $\mathcal{S}_{V}^{d-1}$, being the case $\mathcal{S}_{V}^{1}$ trivial.

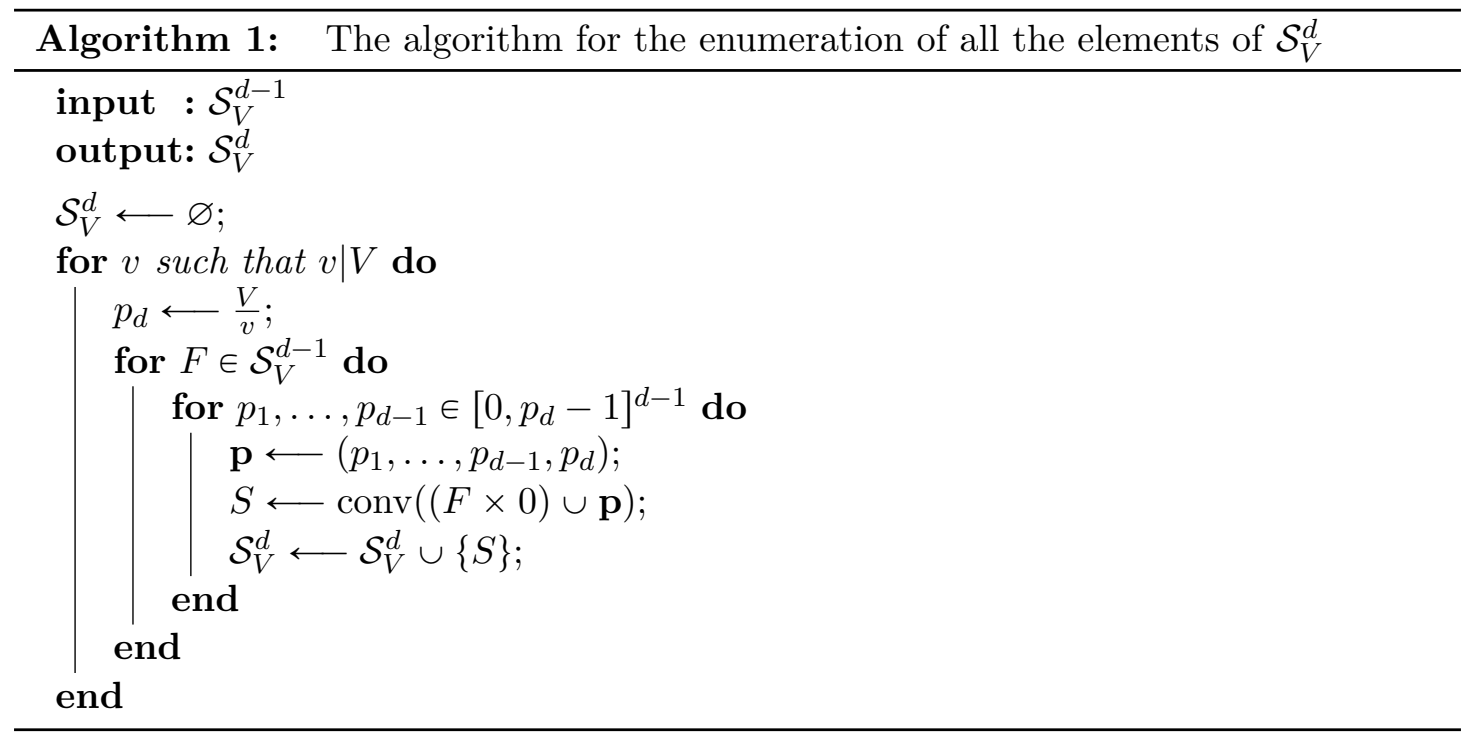

We now discuss the implementation for the complete enumeration of the elements of $\mathcal{P}_{V}^{d}$. We denote by $\mathcal{P}_{\leqslant K}^{d}$ the set of all $d$-dimensional lattice polytopes of normalized volume at most $K$, i.e. $\mathcal{P}_{\leqslant K}^{d}:=\bigcup_{V=1}^{K} \mathcal{P}_{V}^{d}$. Similarly, we set $\mathcal{S}_{\leqslant K}^{d}:=\bigcup_{V=1}^{K} \mathcal{S}_{V}^{d}$. The algorithm works as follows. The simplices of $\mathcal{S}_{\leq K}^{d}$ are used as starting objects for the enumeration. The possible volume vectors of point configuration of cardinality $d+2$ are then calculated and used to iteratively add new vertices to the simplex. This is possible because the volume vector of a point configurations with $d+2$ points encodes the unique affine dependence among them (Lemma 2.4). In order to optimize the implementation, the volume vectors have to be chosen carefully. We use the sign-changed definition of 
the volume vector given in (1). We denote by $\mathcal{W}_{K}^{d}$ the set

$$
\mathcal{W}_{K}^{d}:=\left\{\left(w_{1}, \ldots, w_{d+2}\right) \in[-K, K]^{d+2}: \sum_{w_{i}>0} w_{i}=-\sum_{w_{i}<0} w_{i}<K\right\} \cap \mathbb{Z}^{d+2} .
$$

It contains all the possible volume vectors of point configurations of $d+2$ points whose convex hull has normalised volume at most $K$. Once a simplex $S$ is fixed, we can add new vertices to it using only the volume vectors having $\operatorname{Vol}(S)$ as the first entry. To make the computation even faster one can assume that $\operatorname{Vol}(S)$ is, in absolute value, the highest entry in the volume vector. This means that each polytope would be built starting only from the biggest simplex it contains. Using the volume vectors to determine the growing step of the classification algorithm also provides a handy way to deal with symmetries. Algorithm 2 requires $\mathcal{S}_{\leqslant K}^{d}$ and $\mathcal{W}_{K}^{d}$ as inputs, and returns $\mathcal{P}_{\leqslant K}^{d}$ as output. $\mathcal{S}_{\leqslant K}^{d}$ is obtained by iterating Algorithm 1 for values of $V$ ranging from 1 to $K$, while $\mathcal{W}_{K}^{d}$ can be trivially computed. Given a simplex $S=\operatorname{conv}\left(\mathbf{v}_{0}, \ldots, \mathbf{v}_{d}\right) \in \mathcal{S}_{\leqslant K}^{d}$ and a volume vector $w=\left(w_{1}, \ldots, w_{d+2}\right) \in \mathcal{W}_{K}^{d}$ such that $w_{d+2}=\operatorname{Vol}(S)$, we define $\mathbf{p}_{S, w}$ to be the point of $\mathbb{R}^{d}$ such that $w$ is the volume vector of the point configuration $\left\{\mathbf{v}_{0}, \ldots, \mathbf{v}_{d}, \mathbf{p}_{S, w}\right\}$. Thanks to Lemma 2.4. $\mathbf{p}_{S, w}$ is uniquely determined, indeed

$$
\mathbf{p}_{S, w}=\frac{-\sum_{i=0}^{d} w_{i+1} \mathbf{v}_{i}}{\operatorname{Vol}(S)}
$$

Note that, in general, $\mathbf{p}_{S, w}$ is not a lattice point. At every iteration of Algorithm 2, all the points $\mathbf{p}_{S, w}$ that are lattice points are stored in a temporary variable $\mathcal{X}_{S}$. After that, the elements of $\mathcal{X}_{S}$ are used to "grow" $S$ in all the possible ways. This is done in the second part of the main loop. A variable called $s$ is used to count how many iterations the growing process needs. One can think to $s$ as the variable counting the size of the lattice polytopes, i.e. their number of lattice points. In particular, at every iteration over $s$, only the lattice polytopes of size $s$ will be processed and "grown" by adding one lattice point. Consider that this process ramifies and becomes slower, indeed starting from a single simplex, adding different points obviously generates different polytopes. In order to minimize the number of iterations we use some Ehrhart Theory, which guarantees a simple structure for the polytopes for which the number of lattice points is maximal with respect to the volume. This is done via Lemma 4.1. In order to state it correctly, we need some definitions.

Given a $d$-dimensional lattice polytope $P \subset \mathbb{R}^{d}$, we define the lattice pyramid $\operatorname{Pyr}(P)$ as the $(d+1)$-dimensional polytope

$$
\operatorname{Pyr}(P):=\operatorname{conv}(P \times\{0\} \cup\{(0, \ldots, 0,1)\}) \subset \mathbb{R}^{d+1} .
$$

Moreover, we say that a $d$-dimensional lattice polytope $P$ is an exceptional simplex if $P$ can be obtained via the $(d-2)$-fold iterations of the lattice pyramid construction over the second dilation of a unimodular simplex, that is,

$$
P \cong \operatorname{Pyr}(\cdots(\operatorname{Pyr}(\operatorname{conv}((0,0),(2,0),(0,2)))) \cdots) .
$$

We say that a $d$-dimensional lattice polytope $P \subseteq \mathbb{R}^{d}$ is a Lawrence prism with heights $a_{0}, \ldots, a_{d-1}$ if there exist nonnegative integers $a_{0}, \ldots, a_{d-1}$ such that

$$
P \cong \operatorname{conv}\left(\left\{\mathbf{0}, a_{0} \mathbf{e}_{d}, \mathbf{e}_{1}, \mathbf{e}_{1}+a_{1} \mathbf{e}_{d}, \ldots, \mathbf{e}_{d-1}, \mathbf{e}_{d-1}+a_{d-1} \mathbf{e}_{d}\right\}\right),
$$


where $\mathbf{e}_{1}, \ldots, \mathbf{e}_{d}$ denote the standard basis of $\mathbb{R}^{d}$

Lemma 4.1. Let $P$ be a d-dimensional lattice polytope. Then $\left|P \cap \mathbb{Z}^{d}\right| \leqslant d+\operatorname{Vol}(P)$, with equality if and only if $P$ is either an exceptional simplex or a Lawrence prism.

Proof. The first part of the lemma is also known as Blichfeldt's Theorem Bli14, but in this case it can be easily deduced with some basic Ehrhart Theory (see Proposition 8.1). A polytope for which the equality $\left|P \cap \mathbb{Z}^{d}\right|=d+\operatorname{Vol}(P)$ is attained, must have $h^{*}$ polynomial $h_{P}^{*}(t)=1+(\operatorname{Vol}(P)-1) t$, which has degree one. The rest of the statement is exactly the characterization of lattice polytopes having $h^{*}$-polynomial of degree one by Batyrev-Nill [BN07.

Thanks to this lemma, we know that the variable $s$ can range between $\left|S \cap \mathbb{Z}^{d}\right|$ and $K+d-1$. At every iteration over $s$ the algorithm selects all the lattice polytopes of size $s$, it grows them into larger ones, which are then stored into a set $\mathcal{Q}_{S}$. The union of all the $\mathcal{Q}_{S}$ for all the simplices $S \in \mathcal{S}_{\leqslant K}^{d}$ will be the complete list of $d$-dimensional lattice polytopes having volume at most $K$, except possibly some Lawrence prisms. Those are easy to classify and can be added "manually" as the last step of the algorithm.

Beside the optimizations discussed in this section, other small improvements above have been added to the actual implementations of Algorithms 1 and 2 . Such expedients are obvious verifications, such as not trying to add point to lattice polytopes of volume $K$ or not trying to add the same point twice, and are not reported in the pseudocode, in order to keep it essential.

\section{RESUlts AND COMPARISON WITH EXISTING CLASSIFICATIONS}

In this section we discuss the results of the classification, and we compare it with other existing ones. Algorithms 1 and 2 have been implemented in Magma [BCP97] on Intel Core i7-2600 CPU 3.40GHz. The total running time for all the classifications was roughly one year in total.

Note that the implementation can be easily parallelized and run on large clusters, but this was beyond the resources of the author and the aims of the paper. The implementation has therefore been performed in low dimensions (up to six) and finding a compromise between a large enough volume and a fast enough running time. The classifications are therefore far from any kind of computational limit, and, if there will be request, they can easily be pushed forward.

The two-dimensional case of the classification is not of particular interest, as lattice polygons up to 30 interior lattice points have been classified in Cas12 and the hollow ones, i.e. the ones without interior lattice points, can be easily described. Indeed, a hollow lattice polygon can either be the convex hull of two lattice segments in two consecutive lines, or the exceptional simplex. For completeness, and for making comparisons, this case has been computed anyway, but the computation has been stopped after a few hours.

Specifically, we fully enumerate the elements of $\mathcal{S}_{\leqslant K}$ and $\mathcal{P}_{\leqslant K}^{d}$ for the following couples $d$ and $K$ :

- $d=2$ and $K=50$,

- $d=3$ and $K=36$,

- $d=4$ and $K=24$, 


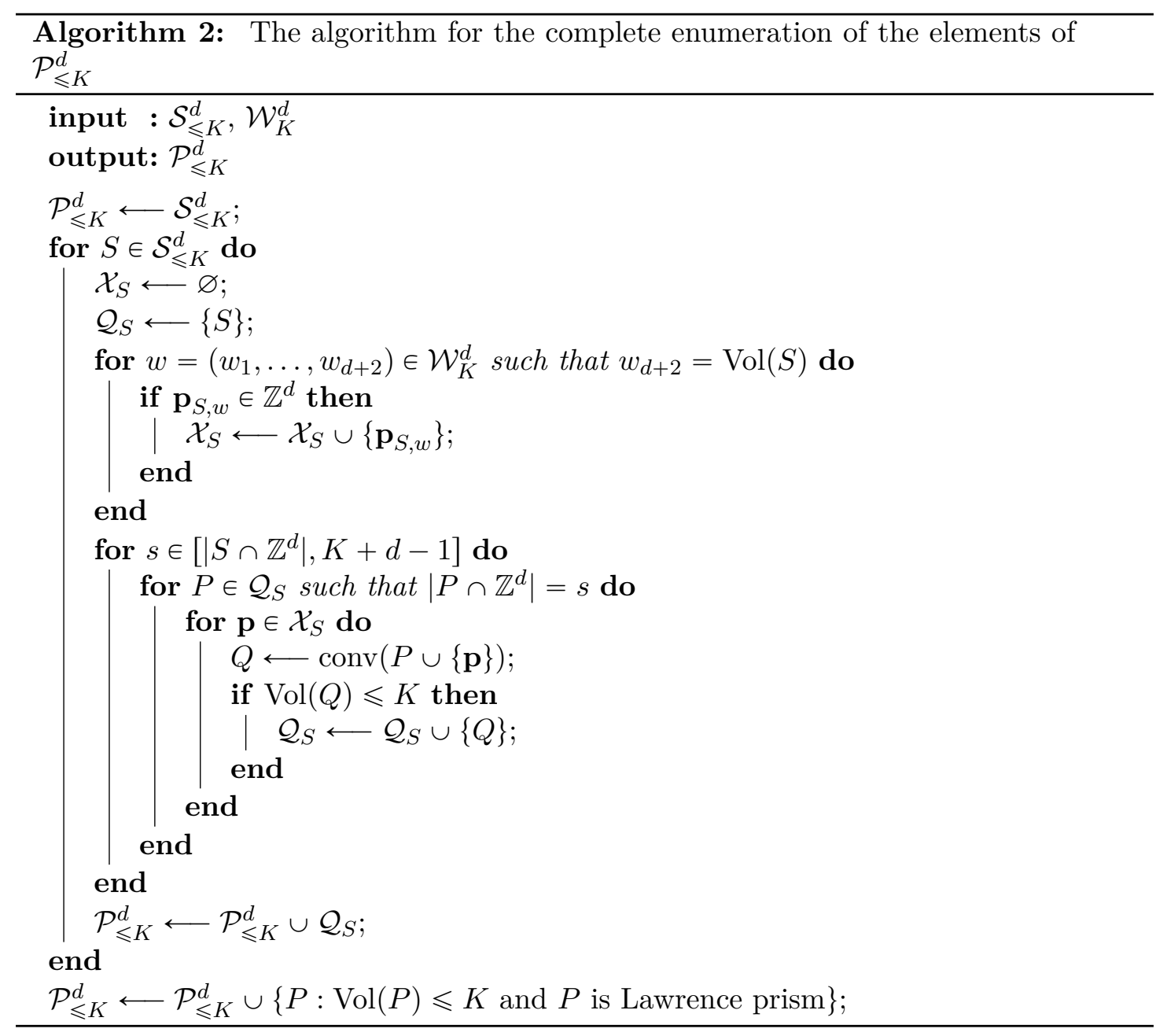

- $d=5$ and $K=20$,

- $d=6$ and $K=16$.

Having in mind applications to Ehrhart Theory, the enumeration of $\mathcal{S}_{\leqslant K}^{3}$ has been performed for $K=1000$ and discussed in Section 7 .

We report here the outcome of the implementation of Algorithm 2, but we discuss applications and implications in the following sections.

Theorem 5.1. Up to unimodular equivalence there are

- 408788 two-dimensional lattice polytopes having volume at most 50.

- 6064034 three-dimensional lattice polytopes having volume at most 36.

- 989694 four-dimensional lattice polytopes having volume at most 24.

- 433273 five-dimensional lattice polytopes having volume at most 20.

- 117084 six-dimensional lattice polytopes having volume at most 16.

Their distribution according to their volume, can be read from the tables in Appendix $C$. 
The resulting database is available at https://github.com/gabrieleballetti/smal 1-lattice-polytopes. An immediate application of a classification result such this, is to double check existing classifications. For example Theorem 5.1 has already been used to correct mistakes in a recent classification result by Hibi and Tsuchiya. In [HT17] they give a characterization for all the lattice polytopes of any dimension, having normalized volume lower than or equal to four. By comparing their results with the classification above it turned out that some polytopes in dimension four and five were missing from their lists. The current version has been corrected with the missing polytopes.

In dimension three our classification has large intersections with several existing ones. In particular we checked that it agrees with:

(1) the classification of three-dimensional lattice polytopes with one interior lattice point Kas10;

(2) the classification of three-dimensional lattice polytopes with two interior lattice points BK16];

(3) the classification of three-dimensional lattice polytopes with width larger than one and having up to eleven lattice points [BS17].

Additionally, our classification fully contains:

(4) the 12 hollow three-dimensional lattice polytopes which are maximal up to inclusion classified in AWW11, AKW17;

(5) the classification of three-dimensional smooth polytopes (see Section 6 for a definition), having up to 16 lattice points, which have been classified in several steps $\mathrm{BHH}^{+} 15$, Lor10, Lun13.

The fact that our classification contains the 12 hollow three-dimensional lattice polytopes which are maximal up to inclusion of [AWW11, AKW17] is not surprising. On the contrary, the classification of three dimensional polytopes was pushed up to normalized volume 36 in order to compare the two classifications. Indeed the largest hollow maximal three-dimensional polytope has volume 36 (this is interesting on its own, as it seems to suggest a "hollow case" of Conjecture 7.2). Anyway, we remark that our classification does not give an independent proof of this fact. On the other hand, it is remarkable how Algorithm 2 seems to be faster than the algorithms to classify smooth polytopes used in $\mathrm{BHH}^{+}$15, Lor10, Lun13.

\section{Smooth POLYTopes}

A natural property in the study of lattice polytopes (especially when it is motivated by toric geometry) is the smoothness. A lattice polytope $P$ in $\mathbb{R}^{d}$ is called smooth if it is simple and if its primitive edge directions at every vertex form a basis of $\mathbb{Z}^{d}$. Sometimes, smooth polytopes are also called Delzant. The word "smooth" comes indeed from the toric varieties realm: a lattice polytope is smooth if and only if the associated projective toric variety is smooth (see [Ful93, Section 2.1]).

The most important open problem regarding smooth polytopes is the so called Oda's conjecture, for which we need to introduce the notion of Integer Decomposition Property. We say that a $d$-dimensional lattice polytope $P$ is $I D P$, or has the Integer Decomposition Property, if for every integer $n \geqslant 1$ and every lattice point $\mathbf{p} \in n P \cap \mathbb{Z}^{d}$ there are lattice points $\mathbf{p}_{1}, \ldots, \mathbf{p}_{n} \in P \cap \mathbb{Z}^{d}$ such that $\mathbf{p}=\mathbf{p}_{1}+\cdots+\mathbf{p}_{n}$. Polytopes having this property 
are often referred to as integrally closed, but one should not confuse them with normal polytopes, which are those which are IDP when considered as lattice polytopes with respect to the lattice affinely spanned by their lattice points. Being IDP is also a very natural property (one can think of it as a discrete counterpart of convexity), which is of interest in algebraic geometry, combinatorics, commutative algebra and optimizations.

In the nineties Oda Oda08 formulated several problem on Minkowski sums of lattice polytopes. One of them is nowadays known in the following form as Oda's Conjecture.

Conjecture 6.1 (Oda's conjecture). Every smooth lattice polytope is IDP.

This is seemingly innocuous statement is actually open even in low dimensions, and in many stronger forms.

In $\left[\mathrm{BHH}^{+} 15\right]$ Bogart et al. prove that for every nonnegative integers $d$ and $n$ there are, modulo unimodular equivalence, there are only finitely many $d$-dimensional smooth polytopes with $n$ lattice points. This can be seen as an evidence for the validity of Conjecture 6.1, as is would follow from it as a corollary (it is indeed easy to verify that there are finitely many IDP polytopes once dimension and number of lattice points are fixed). As an application of their result they classify smooth three-dimensional polytopes having up to 12 lattice points (see also [Lor10]). This was later extended by Lundman [Lun13] who classified all the lattice polytopes having up to 16 lattice points.

Theorem 6.2 ([Lun13, Theorem 1]). Up to unimodular equivalence there exist exactly 103 smooth three-dimensional lattice polytopes $P \subseteq \mathbb{R}^{3}$ such that $\left|P \cap \mathbb{Z}^{3}\right| \leqslant 16$.

The largest polytope in Lundman's classification has normalized volume 23. As a consequence the following result enlarges the current census of "small" three-dimensional polytopes.

Theorem 6.3. Up to unimodular equivalence there exist exactly 1588 smooth threedimensional lattice polytopes $P \subseteq \mathbb{R}^{3}$ such that $\operatorname{Vol}(P) \leqslant 36$. The 103 polytopes having at most 16 lattice points are a subset of them. The distribution of smooth three-dimensional polytopes by their volume is summarized in Table 3.

This highlights how Algorithm 2 seems to be more efficient than the ones used to classify smooth polytopes in $\mathrm{BHH}^{+} 15$, Lor10, Lun13, althought it is not shaped to deal with smooth polytopes. Moreover, Algorithm 2 can be freely used in higher dimension. In particular we can easily obtain results analogous to Theorem 6.3 results up to dimension six.

Theorem 6.4. Up to unimodular equivalence there are

- 1530 two-dimensional smooth polytopes having normalized volume at most 50;

- 1588 three-dimensional smooth polytopes having normalized volume at most 36;

- 738 four-dimensional smooth polytopes having normalized volume at most 24;

- 412 five-dimensional smooth polytopes having normalized volume at most 20;

- 127 six-dimensional smooth polytopes having normalized volume at most 16.

The distribution of the classified smooth polytopes by their volume is summarized in Appendix A. 
Note that, in dimension two, volume and number of lattice points are strictly correlated. It is easy to prove that, for a two-dimensional polytope $P$

$$
\left|P \cap \mathbb{Z}^{2}\right|-2 \leqslant \operatorname{Vol}(P) \leqslant 2\left|P \cap \mathbb{Z}^{2}\right|-5 .
$$

One can see as a consequence of some basic results of Ehrhart Theory, developed in the following section. As a consequence, the classification of two-dimensional smooth polytopes contains all those having up to 27 lattice points. This extends $\mathrm{BHH}^{+} 15$, Theorem 32].

Corollary 6.5. Up to unimodular equivalence there are exactly 458 two-dimensional smooth polytopes having up to 27 lattice points.

Conjecture 6.1 can now be easily verified on the classified polytopes.

Theorem 6.6. Conjecture 6.1 holds for all the smooth polytopes of Theorem 6.4.

By observing Tables 26 in Appendix A one can notice that, in each dimension $d \leqslant 6$, there are only two smooth polytopes of normalized volume lower than or equal to $d$. They are the unimodular simplex $\Delta_{d}$, defined as the convex hull of the origin and the standard basis, which has volume one, and the prism $\Delta_{d-1} \times \Delta_{1}$, which has volume $d$. We now verify that this is always the case, for all $d$. This is indeed a consequence of the combinatorics of simple polytopes.

We are going to use two classical results for simple polytopes. The first is Barnette's Lower Bound Theorem for simple polytopes.

Theorem 6.7 ([Bar71, Theorems 1-2]). Let $P$ be a d-dimensional simple polytope. Denote by $f_{0}$ and $f_{d-1}$ the number of vertices and facets of $P$, respectively. Then,

$$
f_{0} \geqslant(d-1) f_{d-1}-(d+1)(d-2) .
$$

If $d \geqslant 4$, the inequality above is attained with equality only if $P$ of obtained from a simplex via progressive truncations of vertices.

After that we are going to use a description of simple polytopes with $d+2$ facets, that can be found (in a dual version for simplicial polytopes) in Grünbaum's textbook.

Theorem 6.8 ([Grü03, Theorem 6.1.1]). There exist $\left\lfloor\frac{1}{2} d\right\rfloor$ combinatorial types of $d$ dimensional simple polytopes with $d+2$ facets. Those are exactly the products $\Delta_{d-i} \times \Delta_{i}$ for $i=1, \ldots,\left\lfloor\frac{1}{2} d\right\rfloor$.

As a corollary of these result, we describe the combinatorics of simple lattice polytopes having few lattice points.

Lemma 6.9. Let $P$ be a simple $d$-dimensional lattice polytope having at most $3 d-4$ lattice points. Then $P$ is either combinatorially equivalent to the simplex $\Delta_{d}$, or to the prism $\Delta_{1} \times \Delta_{d-1}$.

Proof. $P$ has at most $3 d-4$ vertices. By plugging this number into the inequality of Theorem 6.7, we get an upper bound for the number of facets $f_{d-1}$ that $P$ can have:

$$
f_{d-1} \leqslant d+2 .
$$

Since $f_{d-1}=d+1$ if and only if $P$ is a simplex, we can focus on the case $f_{d-1}=d+2$. By Theorem $6.8 P$ is combinatorially equivalent to the product $\Delta_{i} \times \Delta_{d-i}$, for some 
$1 \leqslant i \leqslant\left\lfloor\frac{1}{2} d\right\rfloor$. In particular, $P$ has $f_{0}=(i+1)(d-i+1)$ vertices. Fixing $d$, this quantity is growing in $i$, so the inequality

$$
f_{0}=(i+1)(d-i+1) \leqslant 3 d-4
$$

is satisfied if and only if $i=1$.

Now, by adding the constraint of being a smooth polytope, we can get a simple description of smooth polytopes having few lattice points.

Theorem 6.10. Let $P$ be a smooth d-dimensional lattice polytope having at most $3 d-4$ lattice points. Then $P$ is a either the unimodular simplex $\Delta_{d}$ or a Lawrence prism with heights $a_{0}, \ldots, a_{d-1}$ with $a_{i} \geqslant 1$ for all $i$, and $\sum_{i=0}^{d-1} a_{i}=2 d-4$.

Proof. If $P$ is a simplex, the statement is trivial. By Lemma 6.9, $P$ is combinatorially equivalent to $\Delta_{1} \times \Delta_{d-1}$, in particular $P$ has two facets $F$ and $F^{\prime}$ which are $(d-$ 1)-dimensional simplices. Since faces of smooth polytopes are smooth, $F$ and $F^{\prime}$ are dilations of $\Delta_{d-1}$. The $t$-th dilation of a $(d-1)$-dimensional simplex has $\left(\begin{array}{c}d+t-1 \\ d-1\end{array}\right)$ lattice points (see e.g. [BR15, Theorem 2.2]), which is lower than or equal to $3 d-4$ only for $t=1$. This proves that both $F$ and $F^{\prime}$ are unimodular equivalent to $\Delta_{d-1}$. Since $P$ is smooth we can assume that

$$
F=\Delta_{d-1}=\operatorname{conv}\left(\mathbf{0}, \mathbf{e}_{1}, \ldots, \mathbf{e}_{d-1}\right),
$$

and that $\mathbf{e}_{d}$ is a lattice point of $P$. Let $E_{0}, \ldots, E_{d-1}$ be the edges of $P$ which are not in $F$ nor in $F^{\prime}$, labeled such that $\mathbf{e}_{i}$ is one vertex of $E_{i}$ for $i=1, \ldots, d-1$, while $\mathbf{0}$ and $\mathbf{e}_{d}$ are in $E_{0}$ ( $\mathbf{e}_{d}$ might not be a vertex). Let moreover $\mathbf{p}_{i}$ be the first lattice point met traveling from $\mathbf{e}_{i}$ along $E_{i}$, for $i=0, \ldots, d-1$, so that $\mathbf{p}_{0}=\mathbf{e}_{d}$. The statement follows by proving that

$$
\operatorname{conv}\left(\mathbf{0}, \mathbf{e}_{1}, \ldots, \mathbf{e}_{d-1}, \mathbf{p}_{0}, \ldots, \mathbf{p}_{d-1}\right)=F \times \Delta_{1} .
$$

By the smoothness assumption, the $\operatorname{simplex} \operatorname{conv}\left(F \cup \mathbf{p}_{i}\right)$ is unimodular for all $i$. This proves that all the $\mathbf{p}_{i}$ 's are at height one, i.e

$$
\tilde{F}:=\operatorname{conv}\left(\mathbf{p}_{0}, \ldots, \mathbf{p}_{d-1}\right)=P \cap\left\{x_{d}=1\right\} .
$$

The combinatorics of $P$ implies that $\tilde{F}$ equals to $t F \times 1$ for a dilation factor $t$, but by the same argument as above, $t=1$.

Having a Lawrence prism structure is very restricting. Lattice points and volume of a Lawrence prisms $P$ are linked by the formula $\left|P \cap \mathbb{Z}^{d}\right|=d+\operatorname{Vol}(P)$ (see Lemma 4.1).

Corollary 6.11. In dimension d the only smooth polytopes having normalized volume at most $d$ are the unimodular simplex $\Delta_{d}$ and the prism $\Delta_{d-1} \times[0,1]$. They have normalized volume 1 and d, respectively.

Lawrence prisms have a very restrictive geometry. It is easy to show (for example using pushing or pulling triangulations) that they have a quadratic triangulation, i.e. a triangulation which is regular unimodular and flag. We refer to [HPPS18] for definitions and terminology about triangulations. The existence of quadratic triangulations for a smooth polytope is a central question in toric geometry as it implies that the associated projective toric variety has a defining ideal generated by quadrics (see [Stu96]). This problem and several of its variations are sometimes known as Bögvad Conjecture. 
Corollary 6.12. Let $P$ be a d-dimensional smooth polytope satisfying one of the following equivalent conditions:

- $\left|P \cap \mathbb{Z}^{d}\right| \leqslant 3 d-4$,

- $\operatorname{Vol}(P) \leqslant 2 d-4$.

Then $P$ has a quadratic triangulation. In particular it is IDP.

Another consequence is the following finiteness result, independent of the dimension.

Corollary 6.13. There are finitely many smooth polytopes of normalized volume $V$, for any fixed integer $V>1$.

By putting together this, together with our classification, we can easily classify all smooth polytopes having normalized volume up to 10 .

Proposition 6.14. Let $P$ be a smooth polytope having normalized volume at most 10 . Then $P$ is either a Lawrence prism, or one of the following 14 polytopes:

(2.a) $\operatorname{conv}\left(\mathbf{0}, 2 \mathbf{e}_{1}, 2 \mathbf{e}_{2}\right)$,

(2.b) $\operatorname{conv}\left(\mathbf{0}, 3 \mathbf{e}_{1}, 3 \mathbf{e}_{2}\right)$,

(2.c) $\operatorname{conv}\left(\mathbf{0}, 2 \mathbf{e}_{1}, 2 \mathbf{e}_{2}, 2 \mathbf{e}_{1}+2 \mathbf{e}_{2}\right)$,

(2.d) $\operatorname{conv}\left(\mathbf{0}, \mathbf{e}_{1}, \mathbf{e}_{2},-2 \mathbf{e}_{1}+\mathbf{e}_{2},-4 \mathbf{e}_{1}+\mathbf{e}_{2}\right)$,

(2.e) $\operatorname{conv}\left(\mathbf{0}, \mathbf{e}_{1}, \mathbf{e}_{2}, 3 \mathbf{e}_{1}+2 \mathbf{e}_{2}, 2 \mathbf{e}_{1}+3 \mathbf{e}_{2}, 3 \mathbf{e}_{1}+3 \mathbf{e}_{2}\right)$,

(2.f) $\operatorname{conv}\left(\mathbf{0}, \mathbf{e}_{1}, \mathbf{e}_{2}, 2 \mathbf{e}_{1}+\mathbf{e}_{2}, \mathbf{e}_{1}+2 \mathbf{e}_{2}, 2 \mathbf{e}_{1}+2 \mathbf{e}_{2}\right)$,

(2.g) $\operatorname{conv}\left(\mathbf{0}, \mathbf{e}_{1}, \mathbf{e}_{2}, 3 \mathbf{e}_{1}+\mathbf{e}_{2}, \mathbf{e}_{1}+2 \mathbf{e}_{2}, 4 \mathbf{e}_{1}+2 \mathbf{e}_{2}\right)$,

(2.h) $\operatorname{conv}\left(\mathbf{0}, \mathbf{e}_{1}, \mathbf{e}_{1}+2 \mathbf{e}_{2},-2 \mathbf{e}_{1}+2 \mathbf{e}_{2}\right)$,

(3.a) $\operatorname{conv}\left(\mathbf{0}, 3 \mathbf{e}_{1}, 3 \mathbf{e}_{2}, 3 \mathbf{e}_{3}\right)$,

(3.b) $\operatorname{conv}\left(\mathbf{0}, \mathbf{e}_{1}, \mathbf{e}_{2}, \mathbf{e}_{1}+\mathbf{e}_{2}, \mathbf{e}_{3}, \mathbf{e}_{1}+\mathbf{e}_{3},-2 \mathbf{e}_{2}+\mathbf{e}_{3}, \mathbf{e}_{1}-2 \mathbf{e}_{2}+\mathbf{e}_{3}\right)$,

(3.c) $\operatorname{conv}\left(\mathbf{0}, \mathbf{e}_{1}, \mathbf{e}_{2}, \mathbf{e}_{3},-2 \mathbf{e}_{2}+\mathbf{e}_{3}, 2 \mathbf{e}_{1}-2 \mathbf{e}_{2}+\mathbf{e}_{3}\right)$,

(3.d) $\operatorname{conv}\left(\mathbf{0}, \mathbf{e}_{1}, \mathbf{e}_{2}, \mathbf{e}_{1}+\mathbf{e}_{2}, \mathbf{e}_{3}, \mathbf{e}_{1}+\mathbf{e}_{3}, \mathbf{e}_{2}+\mathbf{e}_{3}, \mathbf{e}_{1}+\mathbf{e}_{2}+\mathbf{e}_{3}\right)$,

(4.a) $\operatorname{conv}\left(\mathbf{0}, \mathbf{e}_{1}, \mathbf{e}_{2}, \mathbf{e}_{3}, \mathbf{e}_{4}, \mathbf{e}_{1}+\mathbf{e}_{3}, \mathbf{e}_{1}+\mathbf{e}_{4}, \mathbf{e}_{2}+\mathbf{e}_{3}, \mathbf{e}_{2}+\mathbf{e}_{4}\right)$,

(5.a) $\operatorname{conv}\left(\mathbf{0}, \mathbf{e}_{1}, \mathbf{e}_{2}, \mathbf{e}_{3}, \mathbf{e}_{4}, \mathbf{e}_{5}, \mathbf{e}_{1}+\mathbf{e}_{4}, \mathbf{e}_{1}+\mathbf{e}_{5}, \mathbf{e}_{2}+\mathbf{e}_{4}, \mathbf{e}_{2}+\mathbf{e}_{5}, \mathbf{e}_{3}+\mathbf{e}_{4}, \mathbf{e}_{3}+\mathbf{e}_{5}\right)$,

\section{Classifications of 3-Simplices With FeW interior lattice POints}

In this section we show how the classification of polytopes (in particular simplices) with small volume can be used to enumerate all those having a fixed small number of interior lattice points.

It is natural to wonder how large can the volume of a $d$-dimensional polytope $P$ be, when we fix the number of its interior lattice points to be a nonnegative integer $k$. In case $k=0$, i.e. when $P$ is hollow, the answer is clear. One can indeed fit an arbitrarily large $d$-dimensional hollow polytope in the "slab" $[0,1] \times \mathbb{R}^{d-1}$. But in case $k \neq 0$ the answer is different: Hensley Hen83. proved that if $P$ is not hollow, then its volume is bounded by a constant depending only on the dimension $d$ and the number of interior lattice points $k$. In dimension two this was already know, thanks to a sharp bound proven by Scott in 1976 (see Theorem 8.3 in the following section). Hensley's bound has been improved first by Lagarias-Ziegler [LZ91], later by Pikhurko [Pik01], who shown the best currently known bound. 
Theorem 7.1. Let $P$ be a d-dimensional lattice polytope having $k$ interior lattice points, $k \geqslant 1$. Then:

$$
\operatorname{Vol}(P) \leqslant d ! \cdot(8 d)^{d} \cdot 15^{d \cdot 2^{2 d+1}} \cdot k
$$

Although it grows linearly with $k$ (which is the conjectured behaviour, as stated later in Conjecture 7.2), the bound is expected to be very rough. The current largest known volume of a $d$-dimensional lattice polytope having $k$ interior points, $k \geqslant 1$, is given by the $Z P W$ simplex defined as

$$
S_{k}^{d}:=\operatorname{conv}\left(\mathbf{0}, s_{1} \mathbf{e}_{1}, \ldots, s_{d-1} \mathbf{e}_{d-1},(k+1)\left(s_{d}-1\right) \mathbf{e}_{d}\right)
$$

first described by Zaks-Perles-Wills [ZPW82] (hence the namf ${ }^{1}$ ). Here $\left(s_{i}\right)_{i \in \mathbb{Z} \geqslant 1}$ is the Sylvester sequence, defined by the following recursion

$$
s_{1}=2, \quad s_{i}=s_{1} \cdots s_{i-1}+1 .
$$

It has been conjectured that, once $d$ and $k$ are fixed, the ZPW simplex $S_{k}^{d}$ maximises the volume amongst all $k$-point $d$-dimensional polytopes. This conjecture has been explicitly stated in [BK16], but has been already hinted in some of the previously cited works [ZPW82, Hen83, LZ91].

Conjecture 7.2 ([BK16, Conjecture 1.5]). Fix $d \geqslant 3$ and $k \geqslant 1 . A$-dimensional lattice polytope $P$ having $k$ interior lattice points satisfies

$$
\operatorname{Vol}(P) \leqslant(k+1)\left(s_{d}-1\right)^{2} .
$$

With the exception of the case when $d=3, k=1$, this inequality is an equality if and only if $P=S_{k}^{d}$.

The case when $d=3, k=1$ has been addressed in [Kas10: in addition to the ZPW simplex $S_{1}^{3}$, the maximum normalized volume of 72 is also attained by the simplex

$$
\operatorname{conv}((0,0,0),(2,0,0),(0,6,0),(0,0,6)) .
$$

In recent years, Conjecture 7.2 has been proven for several families of lattice polytopes. Explicit classifications [Kas10, BK16] settle the cases $d=3$ and $k \in\{1,2\}$. Averkov-Krümpelmann-Nill AKN15 proved it for simplices with one interior point, while Balletti-Kasprzyk-Nill [BKN16] for reflexive polytopes. Recently Averkov Ave18 proved it for simplices having a facet with one lattice point in its relative interior. In all these families the bound for the volume is sharp as they include the ZPW simplices.

We now use our classification to enumerate all the three-dimensional simplices having few interior lattice points, in this way we will be able to verify Conjecture 7.2 in this additional cases. The idea is to use volume bounds to make sure that our classification contains all lattice polytopes having small numbers of interior points. Note that using the general bounds, even the best ones known (Theorem 7.1) would be futile: one would have to classify all the simplices having up to normalized volume $3.4 \cdot 10^{456}$, in order to be sure that the classification contains all the three-dimensional polytopes with one interior lattice point. Luckily some better bounds are known in special cases.

\footnotetext{
${ }^{1}$ In a personal communication, J. M. Wills explained that the unusual order of the authors of the two pages paper was agreed by the three in order to allow J. Zaks to be the first author of a coauthored paper, at least once.
} 
Theorem 7.3 ([Pik01]). Let $S$ be a three-dimensional lattice simplex having k-interior lattice points, with $k \geqslant 1$. Then

$$
\operatorname{Vol}(S) \leqslant \frac{29791}{352} k \leqslant 85 k .
$$

We now use Algorithm 1 to classify all the elements in $\mathcal{S}_{\leqslant \leqslant 1000}^{3}$.

Proposition 7.4. There are 28015923 three-dimensional simplices having normalized volume at most 1000 .

As an immediate corollary, we are able to fully enumerate the three-dimensional simplices having up to 11 interior lattice points.

Corollary 7.5. All the 3-simplices having up to 11 interior lattice points are in $\mathcal{S}_{\leqslant 1000}^{3}$. Their distribution by number of interior lattice points is summarized in Table 1 .

Note that Corollary 7.5 can be seen as an extension of existing classifications of three-dimensional simplices performed up to two interior lattice points. The 225 threedimensional simplices with one interior lattice points have been enumerated by Borisov and Borisov [BB92, pg. 278], while the 471 with two interior lattice points are classified in [BK16, Theorem 3.4].

TABLE 1. Number of three-dimensional polytopes and simplices having few interior lattice points. The numbers of the polytopes are from [Kas10] and [BK16].

\begin{tabular}{ccc}
\hline $\begin{array}{c}\text { number of } \\
\text { interior points }\end{array}$ & $\begin{array}{c}\text { number of } \\
\text { simplices }\end{array}$ & $\begin{array}{c}\text { number of } \\
\text { polytopes }\end{array}$ \\
\hline 1 & 225 & 674688 \\
2 & 471 & 22673449 \\
3 & 741 & \\
4 & 1206 & \\
5 & 1338 & \\
6 & 2063 & \\
7 & 2191 & \\
8 & 3007 & \\
9 & 3257 & \\
10 & 4216 & \\
11 & 4087 & \\
\hline
\end{tabular}

Conjecture 7.2 can be now verified on the classified objects. In a similar way, we are going to verify that the classify simplices satisfy a conjecture known as Duong Conjecture [Duo08, Conjectures 1-2] It concerns a special class of three-dimensional lattice simplices. We call a lattice polytope clean, if the only lattice points on its boundary are the vertices.

Conjecture 7.6 (Duong Conjecture). Let $S$ be a three-dimensional clean simplex having $k$ interior lattice points, with $k \geqslant 1$. Then

$$
\operatorname{Vol}(S) \leqslant 12 k+8
$$


where the equality is attained if and only if $S$ is the Duong simplex, defined as

$$
D_{k}^{3}:=\operatorname{conv}((0,0,0),(1,0,0),(0,1,0),(3,6 k+1,12 k+8)) .
$$

Theorem 7.7. Conjectures 7.2 and 7.6 hold for three-dimensional simplices having up to 11 interior lattice points.

\section{Conjectural Ehrhart inequalities in dimension three}

In this section we use the classification of three-dimensional polytopes to estimate the behaviour of their $h^{*}$-polynomials. We begin with a quick introduction to Ehrhart Theory, but we refer the interested reader to Beck and Robins' book BR15].

Given a $d$-dimensional lattice polytope $P$ in $\mathbb{R}^{d}$, one can associate a function $t \mapsto$ $\left|t P \cap \mathbb{Z}^{d}\right|$, which counts the number of lattice points in $t P$, the $t$-th dilation of $P$, where $t$ is a positive integer. Ehrhart [Ehr62] proved that this function behaves polynomially, i.e. that there exists a polynomial $\operatorname{ehr}_{P}$, that we call Ehrhart polynomial of $P$, satisfying $\operatorname{ehr}_{P}(t)=\left|t P \cap \mathbb{Z}^{d}\right|$ for $t \geqslant 1$.

Its generating function is the rational function

$$
\sum_{t \geqslant 0} \operatorname{ehr}_{P}(t) z^{t}=\frac{\sum_{i \geqslant 0} h_{i}^{*} z^{i}}{(1-z)^{d+1}}
$$

where $h_{i}^{*}=0$ for any $i \geqslant d+1$. We call the polynomial $h_{P}^{*}(z)=h_{0}^{*}+h_{1}^{*} z+\cdots+h_{s}^{*} z^{s}$ the $h^{*}$-polynomial of $P$ (sometimes also called $\delta$-polynomial), and we set the degree of $P$ to be $s$, the degree of its $h^{*}$-polynomial. In the following we will often identify the $h^{*}$-polynomial with the vector of its coefficients $\left(h_{0}^{*}, h_{1}^{*}, \ldots, h_{d}^{*}\right)$, which is called the $h^{*}$ vector (or $\delta$-vector) of $P$. Some properties of the $h^{*}$-polynomial are well known, and listed in the following proposition.

Proposition 8.1. The coefficients $h_{0}^{*}, h_{1}^{*}, \ldots, h_{d}^{*}$ of the $h^{*}$-polynomial of $P$ are nonnegative integers satisfying the following conditions:

(1) $h_{0}^{*}=1$

(2) $h_{1}^{*}=\left|P \cap \mathbb{Z}^{d}\right|-d-1$;

(3) $h_{d}^{*}=\left|P^{\circ} \cap \mathbb{Z}^{d}\right|$;

(4) $\sum_{i=0}^{d} h_{i}^{*}=\operatorname{Vol}(P)$.

The nonnegativity part of the statement above is a result of Stanley [Sta80], while the rest can be derived from Ehrhart's original approach. Combinatorial interpretations for the other coefficients are possible, but they are not as natural as the ones above. One of the biggest challenges in Ehrhart Theory is to characterize the $h^{*}$-vectors of lattice polytopes.

Question 8.2. For each $d$, which vectors $\left(h_{0}^{*}, h_{1}^{*}, \ldots, h_{d}^{*}\right)$ are $h^{*}$-vectors of some $d$ dimensional lattice polytope?

This question is broadly open even in dimension three. In two dimensions the answer to Question 8.2 was first given by Scott [Sco76].

Theorem 8.3. The vector with integer entries $\left(1, h_{1}^{*}, h_{2}^{*}\right)$ is the $h^{*}$-vector of a twodimensional lattice polytope if and only if one of the following conditions holds: 
(1) $h_{2}^{*}=0$;

(2) $0<h_{2}^{*} \leqslant h_{1}^{*} \leqslant 3 h_{2}^{*}+3$;

(3) $\left(1, h_{1}^{*}, h_{2}^{*}\right)=(1,7,1)$.

The last condition is attained only by the exceptional triangle

$$
2 \Delta_{2}=\operatorname{conv}\left(\mathbf{0}, 2 \mathbf{e}_{1}, 2 \mathbf{e}_{2}\right) .
$$

A generalized version of Theorem 8.3 has been proven in each dimension and for degree two polytopes in Tre10. This has been generalized further to each degree in BH17, showing that there are inequalities for the $h^{*}$-coefficients that are universal, i.e. not depending on dimension and degree.

Some relation among the coefficients of $h^{*}$-polynomial are known, and summarized in the following proposition. The first one can be the deduced directly from Proposition 8.1, the others come from works by Stanley [Sta91] and Hibi Hib94.

Proposition 8.4. Let $P$ be a d-dimensional lattice polytope of degree $s$ with its $h^{*}$-vector $h^{*}(P)=\left(h_{0}^{*}, \ldots, h_{d}^{*}\right)$. Then:

(1) $h_{d}^{*} \leqslant h_{1}^{*}$

(2) $h_{d-1}^{*}+\cdots+h_{d-i}^{*} \leqslant h_{2}^{*}+\cdots+h_{i+1}^{*}$, for $i=1, \ldots, d-1$;

(3) $h_{0}^{*}+\cdots+h_{i} \leqslant h_{s}+\cdots+h_{s-i}$, for $i=0,1, \ldots, s$;

(4) if $h_{d}^{*}>0$, then $h_{1}^{*} \leqslant h_{i}^{*}$, for $i=1,2, \ldots, d-1$.

In recent years Stapledon [Sta09, Sta16] showed the existence of infinite new classes of inequalities, giving explicit formulae in small dimensions.

In dimension three, the known inequalities are far from giving a complete picture of the possible $h^{*}$-vectors. Nevertheless this case has been solved in the case $h_{3}^{*}=0$, i.e. for hollow lattice polytopes. In this case a polytope has degree two, and Treutlein's result [Tre10] gives a necessary condition, while Henk-Tagami [HT09] prove the sufficiency part.

Theorem 8.5 ([Tre10, Theorem 2],[HT09, Proposition 2.10]). The vector with integer entries $\left(1, h_{1}^{*}, h_{2}^{*}, 0\right)$ is the $h^{*}$-vector of a three-dimensional lattice polytope if and only if one of the following conditions holds:

(1) $h_{2}^{*}=0$;

(2) $0 \leqslant h_{1}^{*} \leqslant 3 h_{2}^{*}+3$;

(3) $\left(1, h_{1}^{*}, h_{2}^{*}, 0\right)=(1,7,1,0)$.

The last condition is attained only by the exceptional simplex

$$
\operatorname{conv}\left(\mathbf{0}, 2 \mathbf{e}_{1}, 2 \mathbf{e}_{2}, \mathbf{e}_{3}\right) \text {. }
$$

In the following we use the classification of three-dimensional lattice polytopes to conjecture a set of sharp inequalities describing the behaviour of three-dimensional polytopes with interior points, i.e. the polytopes whose Ehrhart coefficient $h_{3}^{*}$ is nonzero. The immediate way to do this is by plotting the $h^{*}$-vectors of classified polytopes (see Appendix B , and try to understand which inequalities seem to be satisfied.

In what follows, we frequently need to calculate the $h^{*}$-vector of families of lattice simplices depending on a parameter. The following lemma is an example. Its proof outlines how these kind of results can be proven, and similar results will be given without proof in the rest of the section. 
Lemma 8.6. The $Z P W$ simplex

$$
S_{k}^{3}:=\operatorname{conv}((0,0,0),(2,0,0),(0,3,0),(0,0,6 k+6)),
$$

has $h^{*}$-vector $(1,16 k+19,19 k+16, k)$.

Proof. From Proposition 8.1, we can write the $h^{*}$-vector of any three-dimensional lattice polytope $P$ in terms of number of lattice points, number of interior lattice points and volume as

$$
\left(1,\left|P \cap \mathbb{Z}^{d}\right|-4, \operatorname{Vol}(P)-\left|P \cap \mathbb{Z}^{d}\right|-\left|P^{\circ} \cap \mathbb{Z}^{3}\right|+3, \mid P^{\circ} \cap \mathbb{Z}^{3}\right) .
$$

The normalized volume $\operatorname{Vol}\left(S_{k}^{3}\right)$ of $S_{k}^{3}$ can be calculated trivially and equals $36(k+1)$. For the number of interior lattice point we project $S_{k}^{3}$ along $\mathbf{e}_{3}$, and we deduce that they have to be all of the form $(1,1, a)$ for $a \geqslant 1$. Note that $(1,1, k+1)$ can be written as a convex combination of the vertices $(2,0,0),(0,3,0)$ and $(0,0,6 k+6)$ with weights $\frac{1}{2}, \frac{1}{3}, \frac{1}{6}$, respectively, in particular its is on the boundary of $S_{k}^{3}$. Therefore the interior points are all those of the form $(1,1, a)$, with $1 \leqslant a \leqslant k$, and they are exactly $k$. With a similar (and easier) argument, one can count the lattice points in the relative interior of lower dimensional faces of $S_{k}^{3}$. By summing everything up, we get $\left|P \cap \mathbb{Z}^{d}\right|=16 k+23$, and hence the thesis.

We conjecture the following.

Conjecture 8.7. Let $P$ be a three-dimensional lattice polytope having at least one interior lattice point. Then its $h^{*}$-vector $\left(1, h_{1}^{*}, h_{2}^{*}, h_{3}^{*}\right)$ satisfies the following inequalities.
(1) $h_{3}^{*} \leqslant h_{1}^{*}$,
(2) $h_{1}^{*} \leqslant h_{2}^{*}$
(3) $h_{2}^{*} \leqslant 19 h_{3}^{*}+16$
(4) $h_{2}^{*}-h_{1}^{*} \leqslant 9 h_{3}^{*}+9$,
(5) $5 h_{3}^{*} h_{1}^{*}+4 h_{1}^{*}+4 \leqslant 4 h_{3}^{* 2}+4 h_{3}^{*} h_{2}^{*}+5 h_{2}^{*}$.

Moreover, the fourth inequality holds in the stronger form 2

$\left(4^{*}\right) h_{2}^{*}-h_{1}^{*} \leqslant 9 h_{3}^{*}+7$,

unless $P$ is one of the following exceptional cases (listed together with their $h^{*}$-vectors):

(i) $\operatorname{conv}((0,0,0),(1,0,0),(0,1,0),(2,19,25))$,

(ii) $\operatorname{conv}((0,0,0),(1,0,0),(0,1,0),(3,19,28))$,

(iii) $\operatorname{conv}((0,0,0),(1,0,0),(0,1,0),(3,13,19),(1,-2,-3))$,

(iv) $\operatorname{conv}((0,0,0),(1,0,0),(0,1,0),(4,7,11),(-5,-7,-15))$,

(v) $\operatorname{conv}((0,0,0),(1,0,0),(0,1,0),(2,2,3),(-21,-8,-25))$,

(vi) $\operatorname{conv}((0,0,0),(1,0,0),(0,1,0),(5,17,42))$,

(vii) $\operatorname{conv}((0,0,0),(1,0,0),(0,1,0),(5,7,17),(1,-2,-5))$,

(viii) $\operatorname{conv}((0,0,0),(1,0,0),(0,1,0),(7,2,9),(-7,-3,-15))$,

(ix) $\operatorname{conv}((0,0,0),(1,0,0),(0,1,0),(0,0,1),(5,42,-25))$,

(x) $\operatorname{conv}((0,0,0),(1,0,0),(0,1,0),(5,23,45))$,

(xi) $\operatorname{conv}((0,0,0),(1,0,0),(0,1,0),(3 k+3,9 k+8,18 k+15)), \quad(1,4 k+3,13 k+11, k)$

\footnotetext{
2 This inequality was also conjectured by Mónica Blanco and Lukas Katthän (private communication). They expressed it in an equivalent Pick-like form $\operatorname{Vol}(P) \leqslant 2 b+12 i$ where $b$ and $i$ are the number of lattice points of $P$ on its boundary and in its interior respectively.
} 
(xii) $\operatorname{conv}((0,0,0),(1,0,0),(0,1,0),(3,12 k+8,18 k+15)), \quad(1,4 k+3,13 k+11, k)$

where, in the last two cases, $k \in \mathbb{Z}_{\geqslant 1}$. Inequalities (3) and (5) are both attained with equality if and only if, for some $k \geqslant 1, P$ is one of the $Z P W$ simplices

$$
S_{k}^{3}:=\operatorname{conv}((0,0,0),(2,0,0),(0,3,0),(0,0,6 k+6)),
$$

having $h^{*}$-vector $(1,16 k+19,19 k+16, k)$, or the special "almost-ZPW" simplex

$$
\tilde{S}_{1}^{3}:=\operatorname{conv}((0,0,0),(2,0,0),(0,6,0),(0,0,6)),
$$

having $h^{*}$-vector $(1,35,35,1)$.

Inequalities (1) and $\left(4^{*}\right)$ are both attained with equality if and only if, for some $k \geqslant 1$, $P$ is one of the Duong simplices

$$
D_{k}^{3}:=\operatorname{conv}((0,0,0),(1,0,0),(0,1,0),(3,6 k+1,12 k+8))
$$

having $h^{*}$-vector $(1, k, 10 k+7, k)$.

Inequalities (1) and (2) of Conjecture 8.7 are already known to be true, as they are a consequence of Proposition 8.4. Note that Conjecture 8.7 generalizes the threedimensional case of Conjecture 7.2 for the maximal volume of polytopes having interior lattice points, and Conjecture 7.6 (Duong Conjecture). Moreover it generalizes Conjecture 6.1 of [BK16], on the maximal $h^{*}$-coefficients of lattice polytopes with interior lattice points.

Note that inequality (5) of Conjecture 8.7 is non-linear. However, fixing $h_{3}^{*}$ to be larger than one, the inequalities are linear and define a pentagon (see Figure 1). In the special case when $h_{3}^{*}=1$ equalities (2) and (5) coincide. We now give a vertex representation of such a pentagon.

Proposition 8.8. For each $h_{3}^{*} \in \mathbb{Z}_{>1}$, inequalities (1),(2),(3),(4*),(5) define a pentagon in $\mathbb{R}^{4}$ with vertices

$$
\begin{aligned}
\mathbf{v} 1 & :=\left(1, h_{3}^{*}, h_{3}^{*}, h_{3}^{*}\right), \\
\mathbf{v} 2 & :=\left(1,4 h_{3}^{*}+4,4 h_{3}^{*}+4, h_{3}^{*}\right), \\
\mathbf{v} 3 & :=\left(1,16 h_{3}^{*}+19,19 h_{3}^{*}+16, h_{3}^{*}\right), \\
\mathbf{v} 4 & :=\left(1,10 h_{3}^{*}+9,19 h_{3}^{*}+16, h_{3}^{*}\right), \\
\mathbf{v} 5 & :=\left(1, h_{3}^{*}, 10 h_{3}^{*}+7, h_{3}^{*}\right) .
\end{aligned}
$$

Moreover,

- $\mathbf{v}_{1}$ is realized as the $h^{*}$-vector of the polytope

$$
\operatorname{conv}\left((0,0,0),(1,0,0),(0,1,0),\left(3,3 h_{3}^{*}, 3 h_{3}^{*}+1\right)\right),
$$

- $\mathbf{v}_{2}$ is realized as the $h^{*}$-vector of the polytope

$$
\operatorname{conv}\left((0,0,0),(1,0,0),(2,3,0),\left(2,3,3+3 h_{3}^{*}\right)\right),
$$

- $\mathbf{v}_{3}$ is realized as the $h^{*}$-vector of the $Z P W$ simplex $S_{h_{3}^{*}}^{3}$,

- $\mathbf{v}_{5}$ realized as the $h^{*}$-vector of the Duong simplex $D_{h_{3}^{*}}^{3}$. 


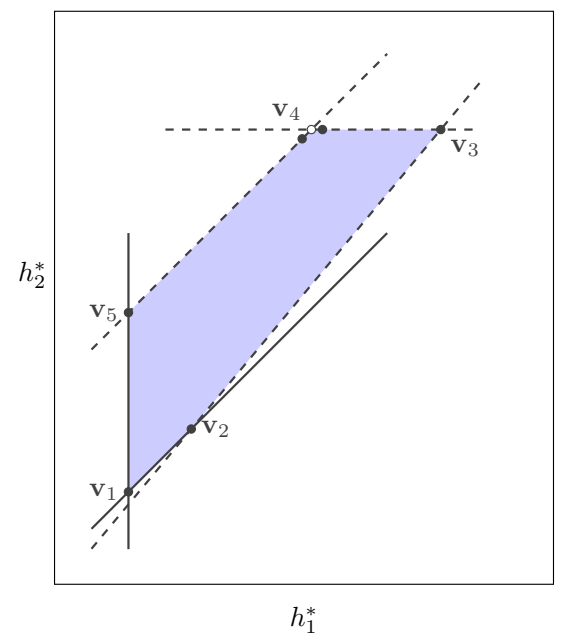

Figure 1. The pentagon defined by Conjecture 8.7, for a fixed $h_{3}^{*}$. The dashed lines represent inequalities (3), $\left(4^{*}\right)$ and (5), which are conjectural. Note that the vertex $\mathbf{v}_{4}$ seems not to be realized as the $h^{*}$-vector of any lattice polytope, anyway there are other realizable $h^{*}$-vector which are very close to it (see Proposition 8.9 and the previous discussion).

Note that we do not have a candidate polytope realizing the vertex $\mathbf{v}_{4}$, for each value of $h_{3}^{*}$. From the data available, it seems like such $h^{*}$-vector is never attained for any number of interior point. Anyway, in order to show that inequalities (3) and $\left(4^{*}\right)$ are actually sharp, we give examples of polytopes having, for each $h_{3}^{*}, h^{*}$-vector "close enough" to $\mathbf{v}_{4}$. We remark that, in this way, there should be an additional inequality in Conjecture 8.7 cutting out the vertex $\mathbf{v}_{4}$, and creating two additional ones. Anyway the distance of the two new vertices from $\mathbf{v}_{4}$ is fixed and does not depend on the value of $h_{3}^{*}$, so we decided not to include it.

Proposition 8.9. For each positive integer value of $h_{3}^{*}$, the $h^{*}$-vectors

- $\left(1,10 h_{3}^{*}+11,19 h_{3}^{*}+16, h_{3}^{*}\right)$,

- $\left(1,10 h_{3}^{*}+9,19 h_{3}^{*}+15, h_{3}^{*}\right)$,

- $\left(1,10 h_{3}^{*}+7,19 h_{3}^{*}+14, h_{3}^{*}\right)$,

are attained, respectively, by the polytopes

- $\operatorname{conv}\left((1,0,0),(2,0,0),(0,1,0),(0,3,0),\left(0,0,6 h_{3}^{*}+5\right),\left(1,0,3 h_{3}^{*}+3\right)\right)$,

- $\operatorname{conv}\left((0,0,0),(1,0,0),(0,1,0),\left(9 h_{3}^{*}+8,6 h_{3}^{*}+5,18 h_{3}^{*}+15\right)\right.$,

$$
\left.\left(12 h_{3}^{*}+10,8 h_{3}^{*}+7,24 h_{3}^{*}+20\right)\right),
$$

- $\operatorname{conv}\left((0,0,0),(1,0,0),(0,1,0),\left(6 h_{3}^{*}+5,3 h_{3}^{*}+3,18 h_{3}^{*}+15\right)\right.$,

$$
\left.\left(8 h_{3}^{*}+5,4 h_{3}^{*}+3,24 h_{3}^{*}+14\right)\right) .
$$

Proof. Using the same technique used in Lemma 8.6. one can prove that the simplices

- $S_{h_{3}^{*}}^{3}$,

- $\operatorname{conv}\left((0,0,0),(1,0,0),(0,1,0),\left(12 h_{3}^{*}+10,6 h_{3}^{*}+6,36 h_{3}^{*}+30\right)\right)$,

- $\operatorname{conv}\left((0,0,0),(1,0,0),(0,1,0),\left(12 h_{3}^{*}+8,6 h_{3}^{*}+5,36 h_{3}^{*}+24\right)\right)$, 
have $h^{*}$-vectors respectively equal to

- $\left(1,16 h_{3}^{*}+19,19 h_{3}^{*}+16, h_{3}^{*}\right)$,

- $\left(1,16 h_{3}^{*}+14,19 h_{3}^{*}+15, h_{3}^{*}\right)$,

- $\left(1,16 h_{3}^{*}+9,19 h_{3}^{*}+14, h_{3}^{*}\right)$.

From this, one can obtain the three polytopes starting from the simplices, and progressively cutting out unimodular simplices. At each cut, $h_{1}^{*}$ drops by one, while $h_{2}^{*}$ stays the same.

As a final observation for this section, we plot heat diagrams of the distribution of $h^{*}$-vectors of three-dimensional lattice polytopes having one and two interior lattice points. From Figure 2 one can note that Conjecture 8.7 seems to describe accurately the behaviour of $h^{*}$-vectors in dimension three, and that most of the $h^{*}$-vectors seems to be in the center of the pentagon.

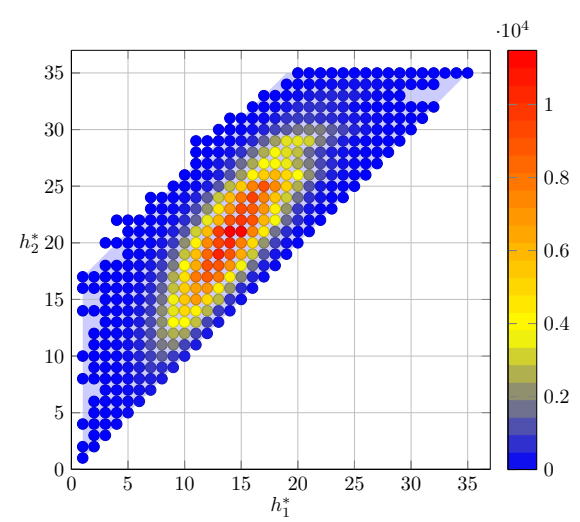

(A) $h_{3}^{*}=1$

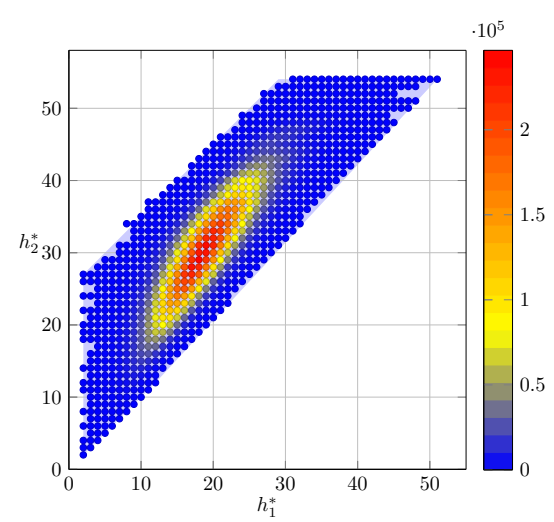

(в) $h_{3}^{*}=2$

FIGURE 2. Heat diagrams for $h^{*}$-vector of three-dimensional polytopes with one and two interior lattice points (from Kas10, BK16]). Each $h^{*}$-vector is coloured according to the number of lattice polytopes attaining it.

\section{Final examples}

In this final section we use the classification to explicitly study how common are some of the most studied properties of lattice polytopes.

In the literature it is possible to find a vast multitude of hierarchies ordering lattice polytopes with a chain of progressively more restricting properties. Having the Integer Decomposition Property (defined in Section 6), plays usually a central role in such a hierarchy, due to its importance in algebraic and optimization contexts. Here we additionally focus on the following properties

Definition 9.1. Let $P \subset \mathbb{R}^{d}$ be a $d$-dimensional lattice polytope. We say that $P$ is spanning if its lattice points affinely span $\mathbb{Z}^{d}$, i.e. if

$$
\mathbb{Z}\left((P-\mathbf{p}) \cap \mathbb{Z}^{d}\right)=\mathbb{Z}^{d}
$$


for any $\mathbf{p} \in P \cap \mathbb{Z}^{d}$. We say that $P$ very ample if for each vertex $\mathbf{v}$ of $P$ the lattice points in the tangent cone $\mathbb{R}_{\geqslant 0}(P-\mathbf{v})$ are sums of lattice points of $P-\mathbf{v}$, i.e if

$$
\mathbb{R}_{\geqslant 0}(P-\mathbf{v}) \cap \mathbb{Z}^{d}=\mathbb{Z}_{\geqslant 0}\left((P-\mathbf{v}) \cap \mathbb{Z}^{d}\right) .
$$

We say that $P$ has a unimodular cover if there exist unimodular lattice simplices $S_{1}, \ldots, S_{n} \subset \mathbb{R}^{d}$ such that $P=S_{1} \cup \cdots \cup S_{n}$. Finally, we say that $P$ has a unimodular triangulation if $P$ admits a triangulation in unimodular lattice simplices.

It is easy to verify that such properties are given in ascending order of restrictiveness, with the IDP property being in the middle, i.e

$$
\underset{\text { triangulation }}{\text { unimodular }} \Rightarrow \begin{gathered}
\text { unimodular } \\
\text { cover }
\end{gathered} \Rightarrow \text { IDP } \Rightarrow \text { very ample } \Rightarrow \text { spanning. }
$$

Notice that in dimension two all these properties are always satisfied by all polytopes. On the other hand, in higher dimensions the opposite inequalities are known to be false. While counterexamples to the last implication (very ampleness implies spanning) are easy to produce, the first example of a very ample but not IDP lattice polytope was given in dimension five by Bruns and Gubeladze [BG02], who later gave an example in dimension three [BG09, Exercise 2.24]. Examples of IDP polytopes not having a unimodular cover have also been given by Bruns and Gubeladze in dimension five [BG99. The first example of a three-dimensional polytope which has a unimodular but does not have a unimodular triangulation has been given by Kantor and Sarkaria [KS03, although the first example, in dimension four, appears in [BGT97].

By looking at the database of three-dimensional polytopes classified in this paper, we can easily find examples of lattice polytopes which are spanning but not very ample, examples of lattice polytopes which are very ample but not IDP, and examples IDP lattice polytopes not admitting a unimodular triangulation. Additionally we can be sure that the following examples are the smallest possible, i.e. those having smallest possible dimension and volume.

Theorem 9.2. The polytope

$$
\operatorname{conv}\left(\left\{\mathbf{0}, \mathbf{e}_{1}, \mathbf{e}_{2}, \mathbf{e}_{3}, \mathbf{e}_{1}+\mathbf{e}_{2}+3 \mathbf{e}_{3}\right\}\right)
$$

is spanning but not very ample. The polytope

$$
\operatorname{conv}\left(\left\{\mathbf{0}, \mathbf{e}_{1}, \mathbf{e}_{2}, \mathbf{e}_{1}+\mathbf{e}_{2}+2 \mathbf{e}_{3}, \mathbf{e}_{1}+\mathbf{e}_{2}+3 \mathbf{e}_{3}, \mathbf{e}_{1}-\mathbf{e}_{3}, \mathbf{e}_{2}-\mathbf{e}_{3}, \mathbf{e}_{3}\right\}\right)
$$

is very ample but not IDP. The polytope

$$
\operatorname{conv}\left(\left\{\mathbf{0}, \mathbf{e}_{1}, \mathbf{e}_{2}, \mathbf{e}_{1}+\mathbf{e}_{2}, \mathbf{e}_{3}, \mathbf{e}_{1}+2 \mathbf{e}_{2}-\mathbf{e}_{3}, 3 \mathbf{e}_{1}+\mathbf{e}_{2}-\mathbf{e}_{3},-2 \mathbf{e}_{1}-\mathbf{e}_{2}+\mathbf{e}_{3}\right\}\right)
$$

has a unimodular cover but does not have a unimodular triangulation. Such examples are those of minimal volume in dimension three.

The last example also appears in [DLST01. No IDP polytope without a unimodular cover has been found in dimesion three, but not all the enumerated three polytopes could be checked, as the algorithm implemented for checking the existence of a unimodular cover is computationally expensive. The presence of such polytopes in higher dimension has already been mentioned, but it makes sense to wonder whether being IDP and having a unimodular cover are equivalent properties in dimension three. 
Question 9.3. Is there a three-dimensional IDP polytope that does not have a unimodular cover?

In this last part of the paper di discuss about properties of the $h^{*}$-vectors of very ample lattice polytopes. We call a sequence $a_{0}, a_{1}, \ldots, a_{n}$ of real numbers unimodal if, for some $0 \leqslant k \leqslant n$,

$$
a_{0} \leqslant \cdots \leqslant a_{k-1} \leqslant a_{k} \geqslant a_{k+1} \geqslant \cdots \geqslant a_{n} .
$$

We call $a_{0}, a_{1}, \ldots, a_{n} \log$-concave if, for all $1 \leqslant i \leqslant n-1$

$$
a_{i-1} a_{i+1} \leqslant a_{i}^{2} .
$$

If all the $a_{i}$ are nonnegative, then log-concavity implies unimodality. It is a long standing open problem (originally posed by Stanley) to understand whether all IDP polytopes have unimodal, or even log-concave, $h^{*}$-vector (see Braun's survey [Bra16]). In [HHJN17, p.39] it is shown that, by relaxing the IDP property to very ampleness, it is possible to lose log-concavity. This is done by giving an example of nine-dimensional very ample (but non IDP) lattice polytope.

By looking at our database it turns out that this kind of examples can be small and exist already in dimension three, as the polytope

$$
\operatorname{conv}\left(\mathbf{0}, e_{1}, e_{2}, e_{3}, e_{1}+e_{3}, e_{2}+e_{3}, e_{1}+e_{2}+16 e_{3}, e_{1}+e_{2}+17 e_{3}\right),
$$

is very ample and has $h^{*}$-vector $(1,4,17,0)$, which is not log-concave. This example can be easily generalized by considering the three-dimensional lattice polytope

$$
Q:=\operatorname{conv}\left(\mathbf{0}, e_{1}, e_{2}, e_{3}, e_{1}+e_{3}, e_{2}+e_{3}, e_{1}+e_{2}+k e_{3}, e_{1}+e_{2}+(k+1) e_{3}\right),
$$

where $k$ is a nonnegative integer. It is easy to verify that $Q$ is a very ample lattice polytope with $h^{*}$-vector $(1,4, k+1,0)$, which, for $k \geqslant 16$, is not log-concave. Note that $Q$ fails to be IDP for $k \geqslant 4$. This kind of constructions are called segmental fibration and have been used in BDGM15] to generate non IDP but very ample polytopes.

\section{REFERENCES}

[AKN15] Gennadiy Averkov, Jan Krümpelmann, and Benjamin Nill. Largest integral simplices with one interior integral point: solution of Hensley's conjecture and related results. Adv. Math., 274:118-166, 2015.

[AKW17] Gennadiy Averkov, Jan Krümpelmann, and Stefan Weltge. Notions of maximality for integral lattice-free polyhedra: the case of dimension three. Math. Oper. Res., 42(4):1035-1062, 2017.

[Ave18] Gennadiy Averkov. Local optimality of Zaks-Perles-Wills simplices. arXiv:1803.04852 [math.CO], 2018.

[AWW11] Gennadiy Averkov, Christian Wagner, and Robert Weismantel. Maximal lattice-free polyhedra: finiteness and an explicit description in dimension three. Math. Oper. Res., 36(4):721$742,2011$.

[Bar71] David W. Barnette. The minimum number of vertices of a simple polytope. Israel J. Math., 10:121-125, 1971.

[Bat81] Victor Batyrev. Toric Fano threefolds. Izv. Akad. Nauk SSSR Ser. Mat., 45(4):704-717, 927, 1981.

[Bat99] Victor Batyrev. On the classification of toric Fano 4-folds. J. Math. Sci. (New York), 94(1):1021-1050, 1999. Algebraic geometry, 9.

[BB92] A. A. Borisov and L. A. Borisov. Singular toric Fano three-folds. Mat. Sb., 183(2):134-141, 1992. 
[BCP97] Wieb Bosma, John Cannon, and Catherine Playoust. The Magma algebra system. I. The user language. J. Symbolic Comput., 24(3-4):235-265, 1997. Computational algebra and number theory (London, 1993).

[BDGM15] Matthias Beck, Jessica Delgado, Joseph Gubeladze, and Mateusz Michałek. Very ample and Koszul segmental fibrations. J. Algebraic Combin., 42(1):165-182, 2015.

[BG99] Winfried Bruns and Joseph Gubeladze. Normality and covering properties of affine semigroups. J. Reine Angew. Math., 510:161-178, 1999.

[BG02] Winfried Bruns and Joseph Gubeladze. Semigroup algebras and discrete geometry. In Geometry of toric varieties, volume 6 of Sémin. Congr., pages 43-127. Soc. Math. France, Paris, 2002.

[BG09] Winfried Bruns and Joseph Gubeladze. Polytopes, rings, and K-theory. Springer Monographs in Mathematics. Springer, Dordrecht, 2009.

[BGT97] Winfried Bruns, Joseph Gubeladze, and Ngô Viêt Trung. Normal polytopes, triangulations, and Koszul algebras. J. Reine Angew. Math., 485:123-160, 1997.

[BH17] Gabriele Balletti and Akihiro Higashitani. Universal inequalities in Ehrhart Theory. arXiv:1703.09600 [math.C0], 2017.

$\left[\mathrm{BHH}^{+} 15\right]$ Tristram Bogart, Christian Haase, Milena Hering, Benjamin Lorenz, Benjamin Nill, Andreas Paffenholz, Günter Rote, Francisco Santos, and Hal Schenck. Finitely many smooth $d$-polytopes with $n$ lattice points. Israel J. Math., 207(1):301-329, 2015.

[BK16] Gabriele Balletti and Alexander M. Kasprzyk. Three-dimensional lattice polytopes with two interior lattice points. arXiv:1612.08918 [math.C0], 2016.

[BKN16] Gabriele Balletti, Alexander M. Kasprzyk, and Benjamin Nill. On the maximum dual volume of a canonical fano polytope. arXiv:1611.02455 [math.CO], 2016.

[Bli14] H. F. Blichfeldt. A new principle in the geometry of numbers, with some applications. Trans. Amer. Math. Soc., 15(3):227-235, 1914.

[BN07] Victor Batyrev and Benjamin Nill. Multiples of lattice polytopes without interior lattice points. Mosc. Math. J., 7(2):195-207, 349, 2007.

[BR15] Matthias Beck and Sinai Robins. Computing the continuous discretely. Undergraduate Texts in Mathematics. Springer, New York, second edition, 2015.

[Bra16] Benjamin Braun. Unimodality problems in Ehrhart theory. In Recent trends in combinatorics, volume 159 of IMA Vol. Math. Appl., pages 687-711. Springer, [Cham], 2016.

[BS16a] Mónica Blanco and Francisco Santos. Lattice 3-polytopes with few lattice points. SIAM J. Discrete Math., 30(2):669-686, 2016.

[BS16b] Mónica Blanco and Francisco Santos. Lattice 3-polytopes with six lattice points. SIAM J. Discrete Math., 30(2):687-717, 2016.

[BS17] Mónica Blanco and Francisco Santos. Enumeration of lattice 3-polytopes by their number of lattice points. Discrete $\&$ Computational Geometry, Sep 2017.

[Cas12] Wouter Castryck. Moving out the edges of a lattice polygon. Discrete Comput. Geom., 47(3):496-518, 2012.

[DLRS10] Jesús A. De Loera, Jörg Rambau, and Francisco Santos. Triangulations, volume 25 of Algorithms and Computation in Mathematics. Springer-Verlag, Berlin, 2010. Structures for algorithms and applications.

[DLST01] Jesús A. De Loera, Francisco Santos, and Fumihiko Takeuchi. Extremal properties for dissections of convex 3-polytopes. SIAM J. Discrete Math., 14(2):143-161, 2001.

[Duo08] Han Duong. Minimal volume k-point lattice d-simplices. arXiv:0804.2910 [math.C0], 2008.

[Ehr62] Eugène Ehrhart. Sur les polyèdres rationnels homothétiques à $n$ dimensions. C. R. Acad. Sci. Paris, 254:616-618, 1962.

[Ful93] William Fulton. Introduction to toric varieties, volume 131 of Annals of Mathematics Studies. Princeton University Press, Princeton, NJ, 1993. The William H. Roever Lectures in Geometry.

[Grü03] Branko Grünbaum. Convex polytopes, volume 221 of Graduate Texts in Mathematics. Springer-Verlag, New York, second edition, 2003. Prepared and with a preface by Volker Kaibel, Victor Klee and Günter M. Ziegler. 
[Hen83] Douglas Hensley. Lattice vertex polytopes with interior lattice points. Pacific J. Math., 105(1):183-191, 1983.

[HHJN17] Takayuki Hibi, Akihiro Higashitani, Katharina Jochemko, and Benjamin Nill. MiniWorkshop: Lattice Polytopes: Methods, Advances, Applications. Oberwolfach Rep., 14(3):2659-2701, 2017.

[Hib94] Takayuki Hibi. A lower bound theorem for Ehrhart polynomials of convex polytopes. Adv. Math., 105(2):162-165, 1994.

[HPPS18] Christian Haase, Andreas Paffenholz, Lindsay C. Piechnik, and Francisco Santos. Existence of unimodular triangulations - positive results. Mem. Amer. Math. Soc., 2018+. to appear.

[HT09] Martin Henk and Makoto Tagami. Lower bounds on the coefficients of Ehrhart polynomials. European J. Combin., 30(1):70-83, 2009.

[HT17] Takayuki Hibi and Akiyoshi Tsuchiya. Classification of lattice polytopes with small volumes. arXiv:1708.00413 [math.C0], 2017.

[Kas10] Alexander M. Kasprzyk. Canonical toric Fano threefolds. Canad. J. Math., 62(6):1293-1309, 2010.

[KN09] Maximilian Kreuzer and Benjamin Nill. Classification of toric Fano 5-folds. Adv. Geom. $9(1): 85-97,2009$.

[KS98] Maximilian Kreuzer and Harald Skarke. Classification of reflexive polyhedra in three dimensions. Adv. Theor. Math. Phys., 2(4):853-871, 1998.

[KS00] Maximilian Kreuzer and Harald Skarke. Complete classification of reflexive polyhedra in four dimensions. Adv. Theor. Math. Phys., 4(6):1209-1230, 2000.

[KS03] J.-M. Kantor and K. S. Sarkaria. On primitive subdivisions of an elementary tetrahedron. Pacific J. Math., 211(1):123-155, 2003.

[Lor10] Benjamin Lorenz. Classification of smooth lattice polytopes with few lattice points. arXiv:1001.0514 [math.CO], 2010.

[LP08] Benjamin Lorenz and Andreas Paffenholz. Smooth reflexive polytopes up to dimension 9. http://polymake.org/polytopes/paffenholz/www/fano.html, 2008.

[Lun13] Anders Lundman. A classification of smooth convex 3-polytopes with at most 16 lattice points. J. Algebraic Combin., 37(1):139-165, 2013.

[LZ91] Jeffrey C. Lagarias and Günter M. Ziegler. Bounds for lattice polytopes containing a fixed number of interior points in a sublattice. Canad. J. Math., 43(5):1022-1035, 1991.

[Øb07] Mikkel Øbro. An algorithm for the classification of smooth Fano polytopes. arXiv: 0704.0049 [math.CD], 2007.

[Oda08] Tadao Oda. Problems on minkowski sums of convex lattice polytopes. arXiv:0812.1418 [math.AG], 2008.

[Pik01] Oleg Pikhurko. Lattice points in lattice polytopes. Mathematika, 48(1-2):15-24 (2003), 2001.

[Sat00] Hiroshi Sato. Toward the classification of higher-dimensional toric Fano varieties. Tohoku Math. J. (2), 52(3):383-413, 2000.

[Sco76] P. R. Scott. On convex lattice polygons. Bull. Austral. Math. Soc., 15(3):395-399, 1976.

[Sta80] Richard P. Stanley. Decompositions of rational convex polytopes. Ann. Discrete Math. 6:333-342, 1980. Combinatorial mathematics, optimal designs and their applications (Proc. Sympos. Combin. Math. and Optimal Design, Colorado State Univ., Fort Collins, Colo., 1978).

[Sta91] Richard P. Stanley. On the Hilbert function of a graded Cohen-Macaulay domain. J. Pure Appl. Algebra, 73(3):307-314, 1991.

[Sta09] Alan Stapledon. Inequalities and Ehrhart $\delta$-vectors. Trans. Amer. Math. Soc., 361(10):56155626, 2009.

[Sta16] Alan Stapledon. Additive number theory and inequalities in Ehrhart theory. Int. Math. Res. Not. IMRN, (5):1497-1540, 2016.

[Stu96] Bernd Sturmfels. Gröbner bases and convex polytopes, volume 8 of University Lecture Series. American Mathematical Society, Providence, RI, 1996.

[Tre10] Jaron Treutlein. Lattice polytopes of degree 2. J. Combin. Theory Ser. A, 117(3):354-360, 2010 . 
[WW82] Keiichi Watanabe and Masayuki Watanabe. The classification of Fano 3-folds with torus embeddings. Tokyo J. Math., 5(1):37-48, 1982.

[ZPW82] J. Zaks, M. A. Perles, and J. M. Wilks. On lattice polytopes having interior lattice points. Elem. Math., 37(2):44-46, 1982 
Appendix A. Smooth polytopes

TABle 2. Distribution of two-dimensional smooth polytopes by their normalized volume.

\begin{tabular}{|c|c|c|c|c|c|}
\hline volume & $\begin{array}{l}\text { smooth } \\
\text { polytopes }\end{array}$ & volume & $\begin{array}{l}\text { smooth } \\
\text { polytopes }\end{array}$ & volume & $\begin{array}{c}\text { smooth } \\
\text { polytopes }\end{array}$ \\
\hline 1 & 1 & 18 & 15 & 35 & 42 \\
\hline 2 & 1 & 19 & 16 & 36 & 41 \\
\hline 3 & 1 & 20 & 18 & 37 & 35 \\
\hline 4 & 3 & 21 & 13 & 38 & 60 \\
\hline 5 & 2 & 22 & 23 & 39 & 53 \\
\hline 6 & 4 & 23 & 21 & 40 & 56 \\
\hline 7 & 4 & 24 & 24 & 41 & 41 \\
\hline 8 & 6 & 25 & 19 & 42 & 63 \\
\hline 9 & 5 & 26 & 26 & 43 & 61 \\
\hline 10 & 7 & 27 & 25 & 44 & 62 \\
\hline 11 & 7 & 28 & 30 & 45 & 61 \\
\hline 12 & 9 & 29 & 22 & 46 & 91 \\
\hline 13 & 7 & 30 & 39 & 47 & 66 \\
\hline 14 & 12 & 31 & 34 & 48 & 72 \\
\hline 15 & 12 & 32 & 34 & 49 & 78 \\
\hline 16 & 15 & 33 & 27 & 50 & 111 \\
\hline 17 & 9 & 34 & 46 & & \\
\hline
\end{tabular}

TABLE 3. Distribution of three-dimensional smooth polytopes by their normalized volume.

\begin{tabular}{|c|c|c|c|c|c|}
\hline volume & $\begin{array}{l}\text { smooth } \\
\text { polytopes }\end{array}$ & volume & $\begin{array}{l}\text { smooth } \\
\text { polytopes }\end{array}$ & volume & $\begin{array}{l}\text { smooth } \\
\text { polytopes }\end{array}$ \\
\hline 1 & 1 & 13 & 16 & 25 & 56 \\
\hline 2 & 0 & 14 & 17 & 26 & 63 \\
\hline 3 & 1 & 15 & 22 & 27 & 79 \\
\hline 4 & 1 & 16 & 22 & 28 & 72 \\
\hline 5 & 2 & 17 & 25 & 29 & 74 \\
\hline 6 & 4 & 18 & 36 & 30 & 103 \\
\hline 7 & 5 & 19 & 33 & 31 & 89 \\
\hline 8 & 6 & 20 & 35 & 32 & 92 \\
\hline 9 & 8 & 21 & 47 & 33 & 115 \\
\hline 10 & 8 & 22 & 43 & 34 & 109 \\
\hline 11 & 10 & 23 & 48 & 35 & 113 \\
\hline 12 & 16 & 24 & 66 & 36 & 151 \\
\hline
\end{tabular}


TABLE 4. Distribution of four-dimensional smooth polytopes by their normalized volume.

\begin{tabular}{|c|c|c|c|c|c|}
\hline volume & $\begin{array}{l}\text { smooth } \\
\text { polytopes }\end{array}$ & volume & $\begin{array}{c}\text { smooth } \\
\text { polytopes }\end{array}$ & volume & $\begin{array}{c}\text { smooth } \\
\text { polytopes }\end{array}$ \\
\hline 1 & 1 & 9 & 6 & 17 & 40 \\
\hline 2 & 0 & 10 & 9 & 18 & 49 \\
\hline 3 & 0 & 11 & 12 & 19 & 54 \\
\hline 4 & 1 & 12 & 16 & 20 & 66 \\
\hline 5 & 1 & 13 & 18 & 21 & 73 \\
\hline 6 & 3 & 14 & 23 & 22 & 86 \\
\hline 7 & 3 & 15 & 28 & 23 & 94 \\
\hline 8 & 5 & 16 & 36 & 24 & 114 \\
\hline
\end{tabular}

TABLE 5. Distribution of five-dimensional smooth polytopes by their normalized volume.

\begin{tabular}{|c|c|c|c|c|c|}
\hline volume & $\begin{array}{l}\text { smooth } \\
\text { polytopes }\end{array}$ & volume & $\begin{array}{l}\text { smooth } \\
\text { polytopes }\end{array}$ & volume & $\begin{array}{l}\text { smooth } \\
\text { polytopes }\end{array}$ \\
\hline 1 & 1 & 8 & 3 & 15 & 30 \\
\hline 2 & 0 & 9 & 5 & 16 & 38 \\
\hline 3 & 0 & 10 & 8 & 17 & 47 \\
\hline 4 & 0 & 11 & 10 & 18 & 57 \\
\hline 5 & 1 & 12 & 13 & 19 & 70 \\
\hline 6 & 1 & 13 & 18 & 20 & 85 \\
\hline 7 & 2 & 14 & 23 & & \\
\hline
\end{tabular}

TABLE 6. Distribution of six-dimensional smooth polytopes by their normalized volume.

\begin{tabular}{|c|c|c|c|c|c|}
\hline volume & $\begin{array}{l}\text { smooth } \\
\text { polytopes }\end{array}$ & volume & $\begin{array}{l}\text { smooth } \\
\text { polytopes }\end{array}$ & volume & $\begin{array}{c}\text { smooth } \\
\text { polytopes }\end{array}$ \\
\hline 1 & 1 & 7 & 1 & 13 & 14 \\
\hline 2 & 0 & 8 & 2 & 14 & 20 \\
\hline 3 & 0 & 9 & 3 & 15 & 27 \\
\hline 4 & 0 & 10 & 5 & 16 & 35 \\
\hline 5 & 0 & 11 & 7 & & \\
\hline 6 & 1 & 12 & 11 & & \\
\hline
\end{tabular}


Appendix B. Plots of $h^{*}$-VeCtors of threE-Dimensional Simplices

FigURE 3. Plots of the distribution of the $h^{*}$-vectors of three-dimensional simplices having up to eleven interior lattice points. The area defined by the inequalities of Conjecture 8.7 is marked in blue.

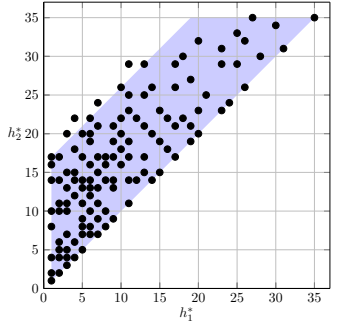

(A) $h_{3}^{*}=1$

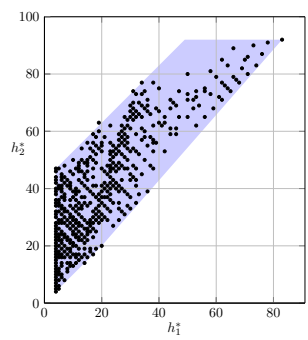

(D) $h_{3}^{*}=4$

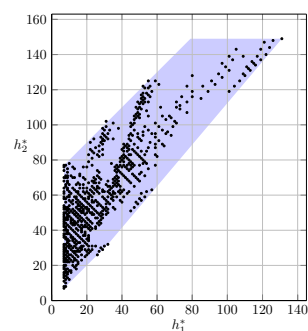

(G) $h_{3}^{*}=7$

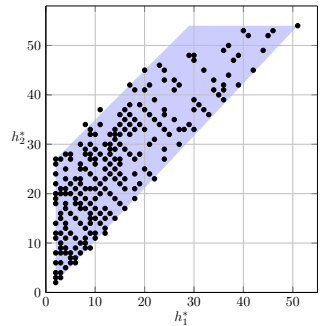

(B) $h_{3}^{*}=2$

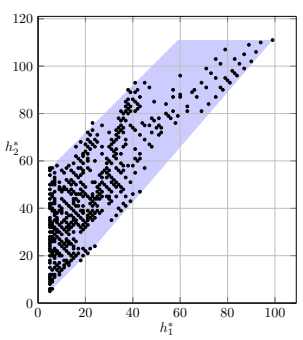

(E) $h_{3}^{*}=5$

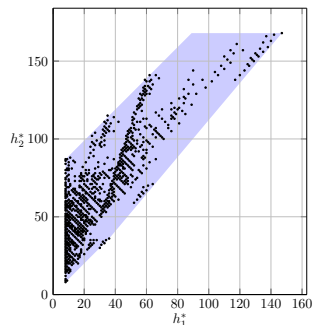

(н) $h_{3}^{*}=8$

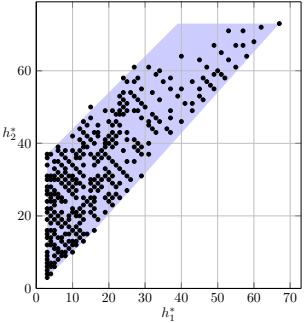

(c) $h_{3}^{*}=3$

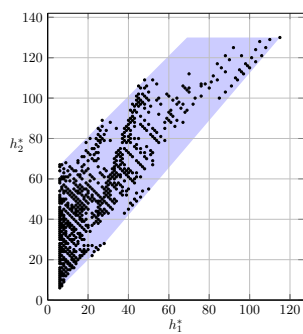

(F) $h_{3}^{*}=6$

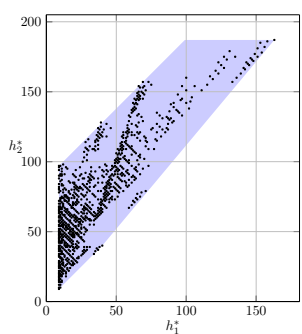

(I) $h_{3}^{*}=9$

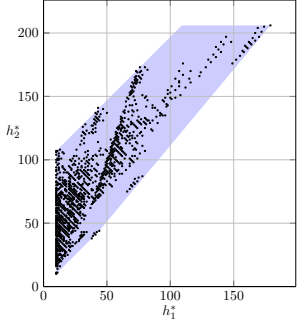

(J) $h_{3}^{*}=10$

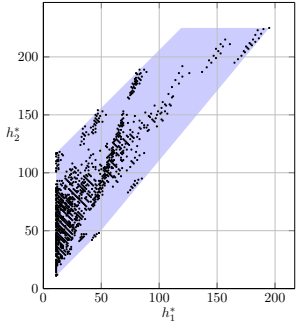

(к) $h_{3}^{*}=11$ 


\section{Appendix C. Frequency of Basic properties of lattice polytopes}

TABLE 7. Number of three-dimensional polytopes (total, spanning, very ample, IDP, having a unimodular cover, having a unimodular triangulation), ordered by their normalized volume

\begin{tabular}{|c|c|c|c|c|c|c|}
\hline Volume & TOT & $\mathrm{SP}$ & VA & IDP & $\mathrm{UC}$ & UT \\
\hline 1 & 1 & 1 & 1 & 1 & 1 & 1 \\
\hline 2 & 3 & 2 & 2 & 2 & 2 & 2 \\
\hline 3 & 6 & 5 & 5 & 5 & 5 & 5 \\
\hline 4 & 17 & 15 & 14 & 14 & 14 & 14 \\
\hline 5 & 19 & 17 & 15 & 15 & 15 & 15 \\
\hline 6 & 54 & 51 & 43 & 43 & 43 & 43 \\
\hline 7 & 59 & 57 & 47 & 47 & 47 & 47 \\
\hline 8 & 154 & 147 & 125 & 125 & 125 & 125 \\
\hline 9 & 181 & 177 & 135 & 135 & 135 & 135 \\
\hline 10 & 368 & 363 & 291 & 290 & 290 & 290 \\
\hline 11 & 414 & 411 & 324 & 323 & 323 & 323 \\
\hline 12 & 961 & 951 & 748 & 746 & 746 & 745 \\
\hline 13 & 1029 & 1025 & 781 & 779 & 779 & 778 \\
\hline 14 & 1929 & 1922 & 1512 & 1506 & 1506 & 1506 \\
\hline 15 & 2409 & 2403 & 1843 & 1837 & 1837 & 1835 \\
\hline 16 & 4254 & 4237 & 3302 & 3292 & 3292 & 3288 \\
\hline 17 & 4983 & 4978 & 3801 & 3787 & & \\
\hline 18 & 8586 & 8574 & 6656 & 6635 & & \\
\hline 19 & 10186 & 10181 & 7809 & 7782 & & \\
\hline 20 & 16708 & 16692 & 13016 & 12971 & & \\
\hline 21 & 20487 & 20479 & 15630 & 15579 & & \\
\hline 22 & 31163 & 31154 & 24167 & 24085 & & \\
\hline 23 & 37779 & 37773 & 29271 & 29171 & & \\
\hline 24 & 58906 & 58876 & 45802 & 45663 & & \\
\hline 25 & 70057 & 70049 & 53907 & 53726 & & \\
\hline 26 & 103117 & 103106 & 80479 & 80225 & & \\
\hline 27 & 126507 & 126495 & 97652 & 97349 & & \\
\hline 28 & 181732 & 181711 & 141923 & 141488 & & \\
\hline 29 & 219325 & 219317 & 170327 & 169816 & & \\
\hline 30 & 311917 & 311898 & 243699 & 242984 & & \\
\hline 31 & 376303 & 376295 & 292843 & 291956 & & \\
\hline 32 & 522559 & 522524 & 409150 & 408010 & & \\
\hline 33 & 636394 & 636382 & 495472 & 494067 & & \\
\hline 34 & 860937 & 860923 & 675187 & 673321 & & \\
\hline 35 & 1043226 & 1043214 & 816386 & 814161 & & \\
\hline 36 & 1411304 & 1411272 & 1106938 & 1104038 & & \\
\hline
\end{tabular}


TABLE 8. Number of four-dimensional polytopes (total, spanning, very ample, IDP, having a unimodular cover, having a unimodular triangulation), ordered by their normalized volume

\begin{tabular}{|c|c|c|c|c|c|c|}
\hline Volume & TOT & SP & VA & IDP & $\mathrm{UC}$ & $\mathrm{UT}$ \\
\hline 1 & 1 & 1 & 1 & 1 & 1 & 1 \\
\hline 2 & 3 & 2 & 2 & 2 & 2 & 2 \\
\hline 3 & 8 & 6 & 6 & 6 & 6 & 6 \\
\hline 4 & 28 & 21 & 19 & 19 & 19 & 19 \\
\hline 5 & 31 & 27 & 21 & 21 & 21 & 21 \\
\hline 6 & 109 & 91 & 71 & 71 & 71 & 71 \\
\hline 7 & 113 & 107 & 74 & 74 & 74 & 74 \\
\hline 8 & 391 & 333 & 242 & 242 & 242 & 242 \\
\hline 9 & 438 & 409 & 255 & 255 & 255 & 255 \\
\hline 10 & 1019 & 956 & 618 & 618 & 618 & 618 \\
\hline 11 & 1109 & 1094 & 664 & 664 & 664 & 664 \\
\hline 12 & 3251 & 2993 & 1851 & 1850 & 1850 & 1849 \\
\hline 13 & 3123 & 3103 & 1762 & 1761 & 1761 & 1760 \\
\hline 14 & 6863 & 6680 & 3921 & 3918 & & \\
\hline 15 & 8506 & 8327 & 4563 & 4560 & & \\
\hline 16 & 17309 & 16681 & 9509 & 9500 & & \\
\hline 17 & 18861 & 18826 & 10074 & 10066 & & \\
\hline 18 & 38061 & 37224 & 20146 & 20125 & & \\
\hline 19 & 42067 & 42023 & 22016 & 21997 & & \\
\hline 20 & 80578 & 79132 & 42297 & 42253 & & \\
\hline 21 & 94373 & 93832 & 47260 & 47214 & & \\
\hline 22 & 158030 & 156975 & 81594 & 81501 & & \\
\hline 23 & 184646 & 184580 & 92530 & 92429 & & \\
\hline 24 & 330776 & 326283 & 165810 & 165631 & & \\
\hline
\end{tabular}

TABle 9. Number of five-dimensional polytopes (total, spanning, very ample, IDP, having a unimodular cover, having a unimodular triangulation), ordered by their normalized volume

\begin{tabular}{|c|c|c|c|c|c|c|}
\hline Volume & TOT & $\mathrm{SP}$ & VA & IDP & $\mathrm{UC}$ & $\mathrm{UT}$ \\
\hline 1 & 1 & 1 & 1 & 1 & 1 & 1 \\
\hline 2 & 4 & 2 & 2 & 2 & 2 & 2 \\
\hline 3 & 10 & 6 & 6 & 6 & 6 & 6 \\
\hline 4 & 38 & 23 & 21 & 21 & 21 & 21 \\
\hline 5 & 42 & 33 & 25 & 25 & 25 & 25 \\
\hline 6 & 169 & 115 & 86 & 86 & 86 & 86 \\
\hline 7 & 163 & 144 & 90 & 90 & 90 & 90 \\
\hline 8 & 659 & 475 & 322 & 322 & 322 & 322 \\
\hline 9 & 707 & 600 & 344 & 344 & 344 & 344 \\
\hline 10 & 1737 & 1465 & 841 & 841 & 841 & 841 \\
\hline
\end{tabular}


Continued from previous page.

\begin{tabular}{|c|c|c|c|c|c|c|}
\hline Volume & TOT & SP & VA & IDP & $\mathrm{UC}$ & $\mathrm{UT}$ \\
\hline 11 & 1743 & 1685 & 869 & 869 & 869 & 869 \\
\hline 12 & 6294 & 5022 & 2791 & 2791 & 2791 & 2790 \\
\hline 13 & 5101 & 5007 & 2392 & 2392 & & \\
\hline 14 & 12640 & 11533 & 5757 & 5756 & & \\
\hline 15 & 15373 & 14315 & 6656 & 6655 & & \\
\hline 16 & 34637 & 30638 & 14873 & 14870 & & \\
\hline 17 & 32858 & 32650 & 14317 & 14314 & & \\
\hline 18 & 77727 & 70953 & 32169 & 32160 & & \\
\hline 19 & 75401 & 75103 & 32282 & 32272 & & \\
\hline 20 & 167969 & 155336 & 68509 & 68488 & & \\
\hline
\end{tabular}

TABLE 10. Number of six-dimensional polytopes (total, spanning, very ample, IDP, having a unimodular cover, having a unimodular triangulation), ordered by their normalized volume

\begin{tabular}{|c|c|c|c|c|c|c|}
\hline Volume & TOT & $\mathrm{SP}$ & $\mathrm{VA}$ & IDP & $\mathrm{UC}$ & UT \\
\hline 1 & 1 & 1 & 1 & 1 & 1 & 1 \\
\hline 2 & 4 & 2 & 2 & 2 & 2 & 2 \\
\hline 3 & 11 & 6 & 6 & 6 & 6 & 6 \\
\hline 4 & 48 & 24 & 22 & 22 & 22 & 22 \\
\hline 5 & 51 & 36 & 27 & 27 & 27 & 27 \\
\hline 6 & 228 & 129 & 94 & 94 & 94 & 94 \\
\hline 7 & 204 & 167 & 97 & 97 & 97 & 97 \\
\hline 8 & 961 & 560 & 362 & 362 & 362 & 362 \\
\hline 9 & 970 & 728 & 392 & 392 & 392 & 392 \\
\hline 10 & 2444 & 1801 & 959 & 959 & 959 & 959 \\
\hline 11 & 2249 & 2092 & 964 & 964 & 964 & 964 \\
\hline 12 & 9872 & 6461 & 3362 & 3362 & & \\
\hline 13 & 6622 & 6334 & 2676 & 2676 & & \\
\hline 14 & 18069 & 14972 & 6684 & 6684 & & \\
\hline 15 & 21837 & 18704 & 7828 & 7828 & & \\
\hline 16 & 53513 & 41025 & 18006 & 18005 & & \\
\hline
\end{tabular}

Department of Mathematics, Stockholm University, SE-106 91 Stockholm, Sweden E-mail address: balletti@math.su.se 